\title{
How unimodular gravity theories differ from general relativity at quantum level
}

\author{
R. Bufalo ${ }^{1,2, \mathrm{a}}$, M. Oksanen ${ }^{1, \mathrm{~b}}$, A. Tureanu ${ }^{1, \mathrm{c}}$ \\ ${ }^{1}$ Department of Physics, University of Helsinki, P.O. Box 64, 00014 Helsinki, Finland \\ ${ }^{2}$ Instituto de Física Teórica (IFT), Universidade Estadual Paulista, Rua Dr. Bento Teobaldo Ferraz 271, \\ Bloco II, São Paulo, SP 01140-070, Brazil
}

Received: 21 May 2015 / Accepted: 16 September 2015 / Published online: 6 October 2015

(C) The Author(s) 2015. This article is published with open access at Springerlink.com

\begin{abstract}
We investigate path integral quantization of two versions of unimodular gravity. First a fully diffeomorphisminvariant theory is analyzed, which does not include a unimodular condition on the metric, while still being equivalent to other unimodular gravity theories at the classical level. The path integral has the same form as in general relativity (GR), except that the cosmological constant is an unspecified value of a variable, and it thus is unrelated to any coupling constant. When the state of the universe is a superposition of vacuum states, the path integral is extended to include an integral over the cosmological constant. Second, we analyze the standard unimodular theory of gravity, where the metric determinant is fixed by a constraint. Its path integral differs from the one of GR in two ways: the metric of spacetime satisfies the unimodular condition only in average over space, and both the Hamiltonian constraint and the associated gauge condition have zero average over space. Finally, the canonical relation between the given unimodular theories of gravity is established.
\end{abstract}

\section{Introduction}

The idea of unimodular gravity is nearly as old as general relativity (GR) itself. Originally, Einstein considered the unimodular condition [1],

$\sqrt{-g}=1$,

as a convenient way to partially fix a coordinate system in GR, which simplifies the calculations in certain situations. Later on, unimodular gravity has also been considered as an alternative theory of gravity closely related to GR, which was

\footnotetext{
a e-mail: rbufalo@ift.unesp.br

b e-mail: markku.oksanen@helsinki.fi

c e-mail: anca.tureanu@helsinki.fi
}

first suggested in [2]. The definition of unimodular gravity is usually based on the invariance under a restricted group of diffeomorphisms that leave the determinant of the metric invariant, so that the determinant of the metric can be set equal to a fixed scalar density $\varepsilon_{0}$,

$\sqrt{-g}=\varepsilon_{0}$,

which provides a fixed volume element in spacetime. We consider a theory based on the condition (1.2) and on the associated restricted group of diffeomorphisms, as the first example of unimodular gravity.

Fully diffeomorphism-invariant extensions of unimodular gravity exist as well, which also involve a condition on the determinant of the metric such that the right-hand side of the condition (1.2) is replaced with a scalar density field. The most prominent theory of this kind is the HenneauxTeitelboim theory [3], where the unimodular condition sets $\sqrt{-g}$ equal to the divergence of a vector density field.

It is well known that classically unimodular gravity produces the same physics as GR with a cosmological constant. The field equation for the metric is either the traceless Einstein equation or, thanks to the Bianchi identity, the Einstein equation with a cosmological constant [4]. The difference is that the cosmological constant of unimodular gravity is a constant of integration, rather than a coupling constant. Since the value of the cosmological constant is unspecified and unrelated to any coupling constant, problems associated with the cosmological constant have been reconsidered (see [5-10] for reviews).

Quantum corrections to the energy-momentum tensor of matter, $T_{\mu \nu}$, which are of the form $C g_{\mu \nu}$, where $C$ is a constant over spacetime, do not contribute to the traceless field equation for the metric in unimodular gravity. In particular, vacuum fluctuations in the trace of the energy-momentum tensor of matter do not affect the metric. This well-known feature of unimodular gravity has been recently revisited via 
an explicit calculation of one-loop corrections [11]. Since a small nonvanishing cosmological constant is required, the full Einstein equation and an associated action need to be considered. There the vacuum corrections are absorbed into the arbitrary cosmological constant, whose value should be specified experimentally. However, this does not solve the cosmological constant problem. Unimodular gravity faces a similar problem with the renormalization or fine tuning of the cosmological constant as GR $[5,7,9,10]$. The expression for the vacuum energy generated by the quantum fluctuations is highly dependent on the details of the effective description, in particular on the chosen Wilsonian cut-off scale [9]. Therefore we do not consider the vacuum energy problem in this paper. Instead we concentrate on the formal differences between the unimodular gravity theories and GR at the quantum level. In other words, our treatment assumes that somehow the observed cosmological constant $\Lambda$ will be stabilized against vacuum corrections.

Predicting or deriving the observed value of the cosmological constant is a hard problem as well. A highly speculative but interesting attempt to address this problem in unimodular gravity has been made in [12-14], where an integral over the cosmological constant was included into the path integral. We will show how this argument can be derived in a straightforward way, when a new action for unimodular gravity is introduced. Problems associated with the given argument are also discussed.

Conventionally, the idea of unimodular gravity has been to impose a condition on the determinant of the metric, e.g., (1.2). In comparison with GR, making the cosmological constant an arbitrary constant of integration can be regarded as the key feature of unimodular gravity. In order to achieve it, however, there is no need to constrain the determinant of the metric. We consider a fully diffeomorphism-invariant theory (see (2.13)), which has recently appeared in the context of gravity with mimetic dark matter [15], where an additional scalar field was also included to describe the mimetic matter. The given theory is no longer unimodular in the sense that there is no condition on the determinant of the metric, but we will establish how the theory is canonically related to the conventional unimodular theories of gravity.

It has been argued that unimodular gravity can offer a new perspective on the problem of time in quantum gravity and cosmology $[4,13,16,17]$. Since the bulk part of the Hamiltonian of unimodular gravity is nonvanishing, and the four-volume provides a cosmological time, an analogy of the Schrödinger equation exists, and hence quantum states of the universe can evolve in terms of a global time. On the other hand, it has been concluded that unimodular gravity cannot solve the problem of time in quantum gravity [18], since the four-volume labels only equivalence classes of hypersurfaces separated by a zero four-volumes.
Since all versions of unimodular gravity must be classically equivalent to GR, quantization of each version of unimodular gravity can be regarded as a potential quantization of GR. Hence it is necessary to understand how the different versions of unimodular gravity differ from each other and from GR at the quantum level. The equivalence of GR and unimodular gravity was recently discussed in [19], concluding that the equivalence can be retained at quantum level when the UV extension of unimodular gravity is performed appropriately. We will see that the form of the path integral depends on which version of unimodular gravity is chosen.

A path integral quantization of the Henneaux-Teitelboim version of unimodular gravity has been considered previously in [14] (see also [20]), where the unimodular condition was shown to be imposed locally in the quantum theory. In this paper, we study the path integral quantization for the two other versions of unimodular gravity discussed above. The results are compared to both GR and the HenneauxTeitelboim theory. In the fully diffeomorphism-invariant theory (see (2.13) for action), the path integral has the same form as the one of GR with a cosmological constant, but the value of $\Lambda$ is an unspecified constant value of a variable. Two approaches regarding the interpretation of the cosmological constant are considered: either (i) the effective value of the cosmological constant is fixed by the physical boundary conditions of the path integral, or (ii) the state of the universe is taken as a superposition of states with different values of $\Lambda$, and consequently the path integral includes an integral over $\Lambda$. In the latter approach, we derive the path integral in the form originally proposed in [12] (see also $[13,14]$ ). In the theory with a fixed metric determinant (see (2.6) for action), the unimodular condition (1.2) is found to be imposed in average over space, but not locally.

In Sect. 2 we present the different actions of unimodular gravity which are relevant for this paper, and we discuss how the (classical) actions are related to each other. Section 3 is devoted to the canonical path integral quantization of the fully diffeomorphism-invariant theory of unimodular gravity. In Sect. 4 the same is achieved for the conventional version of unimodular gravity with a fixed metric determinant. Section 5 establishes the canonical relation of the theories. The results are discussed in Sect. 6.

\section{Three versions of unimodular gravity}

\subsection{Unimodular gravity with a fixed metric determinant}

Conventionally, the field equations of unimodular gravity are obtained from the Einstein-Hilbert action under a restricted variation of the metric $g_{\mu \nu}$ that preserves the determinant of the metric, 


$$
\frac{\delta}{\delta g_{\mu \nu}} \sqrt{-g}=0
$$

where $g=\operatorname{det} g_{\mu \nu}$. Since the metric transforms under an infinitesimal diffeomorphism,

$x^{\prime \mu}(x)=x^{\mu}+\xi^{\mu}(x)$,

as

$\delta_{\xi} g_{\mu \nu}=\nabla_{\mu} \xi_{\nu}+\nabla_{\nu} \xi_{\mu}$,

the unimodular condition (2.1) requires that

$\delta \sqrt{-g}=\frac{1}{2} \sqrt{-g} g^{\mu \nu} \delta g_{\mu \nu}=0$,

i.e.,

$\nabla_{\mu} \xi^{\mu}=0$.

These transformations are often referred to as transverse diffeomorphisms or volume-preserving diffeomorphisms. However, the name transverse diffeomorphisms (TDiff) is sometimes reserved for the transformations that satisfy the noncovariant condition $\partial_{\mu} \xi^{\mu}=0$ [21]. In order to avoid any confusion, we shall refer to the given transformations (2.1)(2.5) as metric determinant-preserving diffeomorphisms.

One way to define unimodular gravity is to introduce the unimodular condition (1.2) into Einstein-Hilbert action as a constraint multiplied by a Lagrange multiplier $\lambda$,

$$
\begin{aligned}
S_{\mathrm{UG}}\left[g_{\mu \nu}, \lambda, \Psi\right]= & \int_{\mathscr{M}} \mathrm{d}^{4} x\left(\frac{\sqrt{-g} R}{\kappa}-\lambda\left(\sqrt{-g}-\varepsilon_{0}\right)\right) \\
& +\frac{2}{\kappa} \oint_{\partial \mathscr{M}} \mathrm{d}^{3} x \sqrt{|\gamma|} \mathscr{K}+S_{\mathrm{m}}\left[g_{\mu \nu}, \Psi\right],
\end{aligned}
$$

where $\varepsilon_{0}$ is a fixed scalar density, such that $\varepsilon_{0} \mathrm{~d}^{4} x$ defines a proper volume element, the gravitational coupling constant is denoted as $\kappa=16 \pi G$, and $S_{\mathrm{m}}$ is the action for the matter fields (denoted collectively by $\Psi$ ) which are coupled to the metric in the same way as in GR. In the surface integral over the boundary $\partial \mathscr{M}$ of spacetime, $\gamma$ is the determinant of the induced metric on $\partial \mathscr{M}$, and $\mathscr{K}$ is the trace of the extrinsic curvature of the boundary. The boundary term is included as in GR, so that the variational principle for the action is well defined without imposing boundary conditions on the derivatives of the metric. ${ }^{1}$ The full diffeomorphism invariance of GR is lost due to the presence of the fixed volume element $\varepsilon_{0} \mathrm{~d}^{4} x$. The action (2.6) is invariant under the metric determinant-preserving diffeomorphisms. We shall refer to the theory defined by (2.6) simply as unimodular gravity (UG).

\footnotetext{
1 When we write about boundary conditions without specifying their nature in the canonical formalism, we refer to both the initial conditions and the conditions on the spatial boundary. Likewise in Lagrangian formalism we refer to conditions on the boundary of spacetime.
}

An unrestricted variation of $g^{\mu \nu}$ gives the Einstein equation

$$
R_{\mu \nu}-\frac{1}{2} R g_{\mu \nu}+\frac{\kappa}{2} \lambda g_{\mu \nu}=\frac{\kappa}{2} T_{\mu \nu} .
$$

The variation of $\lambda$ gives the unimodular condition (1.2). The field equations for matter fields are identical to those of GR. The unimodular condition (1.2) ensures that (2.1) holds. The energy-momentum tensor of matter is defined in (2.7) as usual, $T_{\mu \nu}=-\frac{2}{\sqrt{-g}} \frac{\delta S_{m}}{\delta g^{\mu \nu}}$. We assume that the action for matter is diffeomorphism invariant, so that energy-momentum is conserved, $\nabla^{v} T_{\mu \nu}=0$. Then we take the divergence of (2.7) and obtain

$\nabla_{\mu} \lambda=\nabla^{\nu} T_{\mu \nu}-\frac{2}{\kappa}\left(\nabla^{\nu} R_{\mu \nu}-\frac{1}{2} \nabla_{\mu} R\right)=0$,

where the (contracted) Bianchi identity is used. Thus we see that $\lambda$ is fixed as a constant of integration, which we denote as $\lambda=\frac{2}{\kappa} \Lambda$, where $\Lambda$ is the cosmological constant. Inserting this into the field equation (2.7) gives

$R_{\mu \nu}-\frac{1}{2} R g_{\mu \nu}+\Lambda g_{\mu \nu}=\frac{\kappa}{2} T_{\mu \nu}$.

Compared to GR the only difference is that we are restricted to use coordinate systems that satisfy (1.2). In GR, the condition (1.2) can always be satisfied locally by choosing the inertial coordinates. Then every coordinate system obtained via metric determinant-preserving diffeomorphisms satisfies (1.2) as well.

\subsection{Fully diffeomorphism-invariant unimodular gravity}

Extensions of unimodular gravity with full diffeomorphism invariance have been proposed as well. The most prominent theory is defined by the Henneaux-Teitelboim (HT) action [3] (see [22] regarding the boundary surface term),

$$
\begin{aligned}
& S_{\mathrm{HT}}\left[g_{\mu \nu}, \lambda, \tau^{\mu}, \Psi\right]=\int_{\mathscr{M}} \mathrm{d}^{4} x\left(\frac{\sqrt{-g} R}{\kappa}-\lambda\left(\sqrt{-g}-\partial_{\mu} \tau^{\mu}\right)\right) \\
& +\oint_{\partial \mathscr{M}} \mathrm{d}^{3} x\left(\frac{2}{\kappa} \sqrt{|\gamma|} \mathscr{K}-\lambda r_{\mu} \tau^{\mu}\right)+S_{\mathrm{m}}\left[g_{\mu \nu}, \Psi\right],
\end{aligned}
$$

where $\tau^{\mu}$ is a vector density and $r_{\mu}$ is the outward-pointing unit normal to the boundary $\partial \mathscr{M}$. The field equations consists of the Einstein equation (2.7), the equation for the cosmological constant variable,

$\nabla_{\mu} \lambda=0$,

a (fully diffeomorphism-invariant) unimodular condition,

$\sqrt{-g}=\partial_{\mu} \tau^{\mu}$,

and standard field equations for matter. 
The HT action (2.10) can indeed be derived from the UG action (2.6) via parameterization of the spacetime coordinates [18]. Parametrization of coordinates in a mechanical system is a well known method for obtaining a reparameterization-invariant action (see [23] for a review). Parametrization of field theories was introduced later (see [24] for a description). We treat the coordinates of the action (2.6) as four independent scalar variables $X^{\alpha}(x)$ that depend on the actual coordinates $x^{\mu}$. One can think of this as a transformation $x^{\alpha} \rightarrow X^{\alpha}(x)$. The Einstein-Hilbert and matter parts of the action (2.6) are invariant under such transformation, but the part with a fixed volume element is not invariant, since it transforms as $\int \mathrm{d}^{4} x \varepsilon_{0} \lambda \rightarrow$ $\int \mathrm{d}^{4} x \varepsilon_{0} \lambda\left|\partial_{\mu} X^{\alpha}\right|$, where $\left|\partial_{\mu} X^{\alpha}\right|$ is the Jacobian determinant of the transformation. When we identify a vector density as $\tau^{\mu}=4 ! \varepsilon_{0} \delta_{\alpha}^{[\mu} \delta_{\beta}^{\nu} \delta_{\gamma}^{\rho} \delta_{\delta}^{\sigma]} X^{\alpha} \partial_{\nu} X^{\beta} \partial_{\rho} X^{\gamma} \partial_{\sigma} X^{\delta}$, we obtain the HT action (2.10). It is clear that the HT theory is classically equivalent to the UG theory (2.6). However, differences are expected to arise upon quantization.

We consider an alternative action that is fully diffeomorphism invariant and retains the classical equivalence with the other unimodular theories, in particular with (2.6) and (2.10). The action has been studied in the context of gravity with mimetic dark matter [15], where an additional scalar field was also included to describe the mimetic matter. The action is written (without the scalar field) as

$$
\begin{aligned}
& S_{\mathrm{DUG}}\left[g_{\mu \nu}, \lambda, V^{\mu}, \Psi\right]=\int_{\mathscr{M}} \mathrm{d}^{4} x \sqrt{-g}\left(\frac{R}{\kappa}-\lambda-V^{\mu} \nabla_{\mu} \lambda\right) \\
& +\frac{2}{\kappa} \oint_{\partial \mathscr{M}} \mathrm{d}^{3} x \sqrt{|\gamma|} \mathscr{K}+S_{\mathrm{m}}\left[g_{\mu \nu}, \Psi\right],
\end{aligned}
$$

where the variable $V^{\mu}$ is a vector field. We shall refer to this theory as the fully diffeomorphism-invariant unimodular gravity (DUG). The action (2.13) is arguably the most transparent definition of such a theory. The action (2.13) consists of the Einstein-Hilbert action with a variable cosmological constant $\lambda$, and a constraint term for $\lambda$. The vector field $V^{\mu}$ acts as a Lagrange multiplier that ensures $\nabla_{\mu} \lambda$ is zero in every direction, and thus $\lambda$ is a constant. The field equations consists of the Einstein equation (2.7) for the metric, Eq. (2.11) for the cosmological constant variable $\lambda$, an equation for the auxiliary vector field

$\nabla_{\mu} V^{\mu}=1$

and standard field equations for matter. The unimodular condition on the metric determinant, (1.2) or (2.12), has been replaced with the condition (2.14) on the vector field. The vector field does not contribute to the Einstein equation due to Eq. (2.11). In Sect. 3, we will show how the vector field can be eliminated from the Hamiltonian formulation while the canonical representation of diffeomorphism invariance is retained.

It is obvious that the DUG action (2.13) is closely related to the HT action (2.10). An integration by parts in the term $\int \mathrm{d}^{4} x \lambda \partial_{\mu} \tau^{\mu}$ of the HT action, followed by a replacement of the vector density variable with a vector field variable, $\tau^{\mu}=\sqrt{-g} V^{\mu}$, gives the action (2.13). Hence it is clear that these theories are equivalent classically. However, the path integral for the action (2.13) will be shown to differ from the HT case significantly due to the different choice of variable.

The field equations for both the HT and the DUG theories are invariant under the shift

$T_{\mu \nu} \rightarrow T_{\mu \nu}+C g_{\mu \nu}, \quad \lambda \rightarrow \lambda+C$,

where $C$ is a constant. Hence quantum corrections to the trace of the energy-momentum tensor are absorbed into the variable $\lambda$, whose value is an arbitrary constant. The variable $\lambda$ will be shown to remain constant at quantum level in Sect. 3 .

There exist more versions of unimodular gravity in addition to the three theories discussed above; see, for example, $[14,19]$ for other actions. In this paper we will concentrate on the three theories defined by (2.6), (2.10) and (2.13).

\section{Quantization of the fully diffeomorphism-invariant unimodular gravity}

\subsection{Arnowitt-Deser-Misner decomposition of the action}

Spacetime is assumed to admit a foliation to a union of nonintersecting spacelike hypersurfaces. The hypersurfaces $\Sigma_{t}$ are labeled by a scalar $t$ that is constant across each hypersurface. The future-pointing unit normal to $\Sigma_{t}$ is denoted by $n^{\mu}$. The so-called direction of time vector $t^{\mu}$ satisfies $t^{\mu} \nabla_{\mu} t=1$. The metric $g_{\mu \nu}$ has the signature $(-,+,+,+)$, and hence $n_{\mu} n^{\mu}=-1$. Each hypersurface is described by the induced metric on $\Sigma_{t}$,

$h_{\mu \nu}=g_{\mu \nu}+n_{\mu} n_{\nu}$

and by the extrinsic curvature tensor

$K_{\mu \nu}=\nabla_{\mu} n_{v}+n_{\mu} a_{\nu}$

where we have defined the acceleration vector of Eulerian observers by

$a_{\mu}=n^{\nu} \nabla_{\nu} n_{\mu}$

Now we introduce the Arnowitt-Deser-Misner (ADM) variables. The scalar $t$ is taken as the time coordinate. The unit normal to $\Sigma_{t}$ is written as

$n^{0}=\frac{1}{N}, \quad n^{i}=-\frac{N^{i}}{N}$ 
where $N$ is the lapse variable and $N^{i}$ is the shift vector on $\Sigma_{t}$. Latin indices $(i, j, \ldots)$ range from 1 to 3 . Now the metric takes the form

$g_{00}=-N^{2}+N_{i} N^{i}, \quad g_{0 i}=N_{i}, \quad g_{i j}=h_{i j}$,

where $N_{i}=h_{i j} N^{j}$. The extrinsic curvature is written as

$K_{i j}=\frac{1}{2 N}\left(\partial_{t} h_{i j}-D_{i} N_{j}-D_{j} N_{i}\right)$,

where $D$ is the covariant derivative that is compatible with the metric $h_{i j}$ on $\Sigma_{t}$, and $h^{i j}$ is the inverse metric, $h_{i j} h^{j k}=\delta_{i}{ }^{k}$. The trace of extrinsic curvature is denoted by $K=h^{i j} K_{i j}$.

The action is decomposed as follows. The metric determinant is given by

$\sqrt{-g}=N \sqrt{h}$,

where $h=\operatorname{det} h_{i j}$. The scalar curvature is written as

$R=K_{i j} \mathscr{G}^{i j k l} K_{k l}+{ }^{(3)} R+2 \nabla_{\mu}\left(n^{\mu} K-a^{\mu}\right)$,

where the De Witt metric is defined as

$\mathscr{G}^{i j k l}=\frac{1}{2}\left(h^{i k} h^{j l}+h^{i l} h^{j k}\right)-h^{i j} h^{k l}$

and ${ }^{(3)} R$ is the (intrinsic) scalar curvature of $\Sigma_{t}$. The last term in (3.8) is a total derivative which contributes a boundary term into the action.

The vector field is decomposed into components tangent and normal to $\Sigma_{t}$ as

$V^{\mu}={ }_{\perp} V^{\mu}-n^{\mu} V_{\boldsymbol{n}}$,

where

$\perp V^{\mu}=h_{\nu}^{\mu} V^{\nu}, \quad V_{\boldsymbol{n}}=n_{\mu} V^{\mu}$,

and the projection operator onto $\Sigma_{t}$ is defined as

$h^{\mu}{ }_{v}=\delta^{\mu}{ }_{v}+n^{\mu} n_{v}$.

The gravitational part of the action (2.13) is written in ADM form as

$$
\begin{aligned}
& S_{\mathrm{DUG}}\left[N, N^{i}, h_{i j}, \lambda, V_{\boldsymbol{n}}, V^{i}, \Psi\right]=\int \mathrm{d} t \int_{\Sigma_{t}} \mathrm{~d}^{3} x N \sqrt{h} \\
& \quad \times\left[\frac{1}{\kappa}\left(K_{i j}\left(\mathscr{G}^{i j k l} K_{k l}+{ }^{(3)} R\right)-\lambda+V_{\boldsymbol{n}} \nabla_{n} \lambda-V^{i} \partial_{i} \lambda\right]\right. \\
& \quad+S_{\mathscr{B}}+S_{\mathrm{m}}\left[g_{\mu \nu}, \Psi\right],
\end{aligned}
$$

where we denote $V^{i}={ }_{\perp} V^{i}$ and

$\nabla_{n} \lambda=\frac{1}{N}\left(\partial_{t} \lambda-N^{i} \partial_{i} \lambda\right)$,

and the boundary contribution $S_{\mathscr{B}}$ is given as in GR,

$S_{\mathscr{B}}=\frac{2}{\kappa} \int_{\mathscr{B}} \mathrm{d}^{3} x \sqrt{-\gamma}\left(\mathscr{K}+r_{\mu} n^{\mu} K-r_{\mu} a^{\mu}\right)$,

where $\mathscr{B}$ is the timelike part of the boundary $\partial \mathscr{M}$. The surface $\mathscr{B}$ is foliated to a union of two-dimensional surfaces
$\mathscr{B}_{t}$, which come from the intersection of $\Sigma_{t}$ and $\mathscr{B}$. When the hypersurfaces $\mathscr{B}$ and $\Sigma_{t}$ are orthogonal, the surface term (3.15) can be written as [25]

$S_{\mathscr{B}}=\frac{2}{\kappa} \int \mathrm{d} t \int_{\mathscr{B}_{t}} \mathrm{~d}^{2} x N \sqrt{\sigma}^{(2)} K$,

where $\sigma$ is the determinant of the induced metric on $\mathscr{B}_{t}$, and

${ }^{(2)} K$ is the trace of the extrinsic curvature of $\mathscr{B}_{t}$ in $\Sigma_{t}$.

\subsection{Hamiltonian analysis}

Hamiltonian analysis of unimodular gravity in its different forms has been considered in several papers $[3,4,14,17,18$, $20,26,27]$. Since the action (2.13) differs from the previous theories by lacking a unimodular condition and involving the vector field, we present a detailed Hamiltonian analysis.

\subsubsection{Hamiltonian and constraints}

We shall obtain the Hamiltonian and the full set of constraints for the action (2.13). Here we consider pure gravity, since the matter sector is identical to that of GR, and in the end we include matter into the path integral in Sect. 3.3.

First we introduce the canonical momenta $\pi_{N}, \pi_{i}, \pi^{i j}$, $p_{\lambda}, p_{i}$, and $p_{\boldsymbol{n}}$ conjugate to $N, N^{i}, h_{i j}, \lambda, V^{i}$, and $V_{\boldsymbol{n}}$, respectively. Since the action (3.13) is independent of the time derivatives of the variables $N, N^{i}, V_{\boldsymbol{n}}$, and $V^{i}$, their canonically conjugated momenta are primary constraints:

$\pi_{N} \approx 0, \quad \pi_{i} \approx 0, \quad p_{\boldsymbol{n}} \approx 0, \quad p_{i} \approx 0$.

In addition, the definition of the momentum conjugate to $\lambda$ implies the primary constraint

$\mathscr{C}_{\lambda}=p_{\lambda}-\sqrt{h} V_{\boldsymbol{n}} \approx 0$.

The momentum conjugate to the metric $h_{i j}$ is defined as

$\pi^{i j}=\frac{\sqrt{h}}{\kappa} \mathscr{G}^{i j k l} K_{k l}$.

The Hamiltonian is obtained as

$$
\begin{aligned}
H= & \int_{\Sigma_{t}} \mathrm{~d}^{3} x\left(N \mathscr{H}_{T}+N^{i} \mathscr{H}_{i}+v_{N} \pi_{N}+v_{N}^{i} \pi_{i}+v_{\lambda} \mathscr{C}_{\lambda}\right. \\
& \left.+v_{\boldsymbol{n}} p_{\boldsymbol{n}}+v^{i} p_{i}\right)+H_{\mathscr{B}_{t}},
\end{aligned}
$$

where the so-called super-Hamiltonian and supermomentum are defined as

$\mathscr{H}_{T}=\frac{\kappa}{\sqrt{h}} \pi^{i j} \mathscr{G}_{i j k l} \pi^{k l}-\frac{\sqrt{h}}{\kappa}^{(3)} R+\sqrt{h} \lambda+\sqrt{h} V^{i} \partial_{i} \lambda$

and

$\mathscr{H}_{i}=-2 h_{i j} D_{k} \pi^{j k}+\partial_{i} \lambda p_{\lambda}$, 
respectively, where we introduced the inverse De Witt metric as

$\mathscr{G}_{i j k l}=\frac{1}{2}\left(h_{i k} h_{j l}+h_{i l} h_{j k}\right)-\frac{1}{2} h_{i j} h_{k l}$,

and $v_{N}, v_{N}^{i}, v_{\lambda}, v^{i}, v_{n}$ are unspecified Lagrange multipliers for the primary constraints. Regarding the surface terms, the analysis follows the standard set by [25]. The surface term in the Hamiltonian (3.20) is obtained as

$H_{\mathscr{B}_{t}}=-\frac{2}{\kappa} \int_{\mathscr{B}_{t}} \mathrm{~d}^{2} x N \sqrt{\sigma}^{(2)} K+2 \int_{\mathscr{B}_{t}} \mathrm{~d}^{2} x N^{i} h_{i j} r_{k} \pi^{j k}$

which is the same expression as in GR. The physical Hamiltonian is defined with respect to a chosen reference background as $H_{\text {phys }}=H-H_{\text {ref }}$, where the Hamiltonian of the background is denoted as $H_{\text {ref }}$. The total gravitational energy of the system is the value of the physical Hamiltonian. The surface term (3.24) is given in a generic form that produces the correct expression of total gravitational energy for different reference backgrounds [25].

We must ensure that every constraint is preserved under time evolution that is generated by the Hamiltonian (3.20). The preservation of $\pi_{N} \approx 0$ is ensured by the Hamiltonian constraint

$\mathscr{H}_{T} \approx 0$

and the preservation of $\pi_{i} \approx 0$ is ensured by the momentum constraint

$\mathscr{H}_{i} \approx 0$.

We can extend the momentum constraint (3.22) with a term that is proportional to the primary constraint $p_{n}$ so that the momentum constraint generates spatial diffeomorphisms on $\Sigma_{t}$ for all the variables that are involved in the constraints. ${ }^{2}$ For that reason we redefine

$\mathscr{H}_{i}=-2 h_{i j} D_{k} \pi^{j k}+\partial_{i} \lambda p_{\lambda}+\partial_{i} V_{\boldsymbol{n}} p_{\boldsymbol{n}} \approx 0$.

It is useful to define global (smeared) versions of these constraints for calculational purposes:

$\mathscr{H}_{T}[\xi]=\int_{\Sigma_{t}} \mathrm{~d}^{3} x \xi \mathscr{H}_{T}, \quad \Phi\left[\chi^{i}\right]=\int_{\Sigma_{t}} \mathrm{~d}^{3} x \chi^{i} \mathscr{H}_{i}$,

where $\xi$ and $\chi^{i}$ are functions on $\Sigma_{t}$. The preservation of the constraint $p_{i} \approx 0$,

$\partial_{t} p_{i}=\left\{p_{i}, H\right\} \approx-N \sqrt{h} \partial_{i} \lambda \approx 0$,

is ensured by introducing a new constraint,

$\mathscr{C}_{i}=\partial_{i} \lambda \approx 0$

${ }^{2}$ We do not need to include a generator for the variables $V^{i}$ and $p_{i}$, since the $V^{i}$-dependent term in the Hamiltonian constraint (3.21) is proportional to the constraint (3.30) found below.
This constraint implies that $\lambda$ is a constant across $\Sigma_{t}$. We define the smeared form of $\mathscr{C}_{i}$ as

$\mathscr{C}\left[\chi^{i}\right]=\int_{\Sigma_{t}} \mathrm{~d}^{3} x \chi^{i} \partial_{i} \lambda$

This constraint is included into the Hamiltonian with a Lagrange multiplier as $\mathscr{C}\left[v_{\lambda}^{i}\right]$. The preservation of the constraint $\mathscr{C}_{\lambda} \approx 0$,

$$
\begin{aligned}
\partial_{t} \mathscr{C}_{\lambda} & =\left\{\mathscr{C}_{\lambda}, H\right\} \\
& \approx\left\{\mathscr{C}_{\lambda}, \mathscr{H}_{T}[N]\right\}-\sqrt{h} v_{\boldsymbol{n}}+\left\{\mathscr{C}_{\lambda}, \mathscr{C}\left[v_{\lambda}^{i}\right]\right\} \approx 0
\end{aligned}
$$

is ensured by fixing the Lagrange multiplier $v_{\boldsymbol{n}}$ of the constraint $p_{n}$ as

$v_{\boldsymbol{n}}=-N+\frac{\kappa}{2} N \frac{h_{i j} \pi^{i j}}{\sqrt{h}} V_{\boldsymbol{n}}+\frac{1}{\sqrt{h}} \partial_{i} v_{\lambda}^{i}$

The preservation of the constraint $p_{\boldsymbol{n}} \approx 0$,

$\partial_{t} p_{\boldsymbol{n}}=\left\{p_{\boldsymbol{n}}, H\right\} \approx \sqrt{h} v_{\lambda} \approx 0$

is ensured by fixing the Lagrange multiplier $v_{\lambda}$ of the constraint $\mathscr{C}_{\lambda}$ as

$v_{\lambda}=0$

Since the constraint $\mathscr{C}_{i}$ is included into the Hamiltonian with a Lagrange multiplier, we can simplify the system by redefining $\mathscr{H}_{T}$ without the part that is proportional to $\mathscr{C}_{i}$. Now the total Hamiltonian is written as

$$
\begin{aligned}
H= & \int_{\Sigma_{t}} \mathrm{~d}^{3} x\left(N \mathscr{H}_{T}^{\prime}+N^{i} \mathscr{H}_{i}+v_{N} \pi_{N}+v_{N}^{i} \pi_{i}\right. \\
& \left.+v^{i} p_{i}+v_{\lambda}^{i} \mathscr{C}_{i}^{\prime}\right)+H_{\mathscr{B}_{t}},
\end{aligned}
$$

where we have defined the constraints

$$
\begin{aligned}
& \mathscr{H}_{T}^{\prime}=\mathscr{H}_{T}-p_{\boldsymbol{n}}+\frac{\kappa}{2} \frac{h_{i j} \pi^{i j}}{\sqrt{h}} V_{\boldsymbol{n}} p_{\boldsymbol{n}} \approx 0,
\end{aligned}
$$

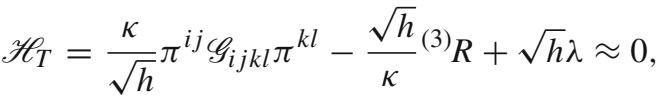

$$
\begin{aligned}
& \mathscr{C}_{i}^{\prime}=\mathscr{C}_{i}-\partial_{i}\left(\frac{p_{\boldsymbol{n}}}{\sqrt{h}}\right) \approx 0,
\end{aligned}
$$

and $v_{N}, v_{N}^{i}, v^{i}, v_{\lambda}^{i}$ are unspecified Lagrange multipliers.

What remains to be established is the preservation of the secondary constraints $\mathscr{H}_{T}, \mathscr{H}_{i}$, and $\mathscr{C}_{i}$ under time evolution. The constraints $\mathscr{H}_{T}, \mathscr{H}_{i}, \mathscr{C}_{i}$ have vanishing Poisson bracket with $p_{n}$. The constraints $\mathscr{H}_{T}, \mathscr{H}_{i}, \mathscr{C}_{i}$ satisfy the following 
algebra:

$$
\begin{aligned}
\left\{\mathscr{H}_{T}[\xi], \mathscr{H}_{T}[\eta]\right\}= & \int_{\Sigma_{t}} \mathrm{~d}^{3} x\left(\xi \partial_{i} \eta-\eta \partial_{i} \xi\right) h^{i j} \\
& \times\left(\mathscr{H}_{j}-p_{\lambda} \mathscr{C}_{j}-\partial_{j} V_{\boldsymbol{n}} p_{\boldsymbol{n}}\right), \\
\left\{\Phi\left[\chi^{i}\right], \mathscr{H}_{T}[\xi]\right\}= & \mathscr{H}_{T}\left[\chi^{i} \partial_{i} \xi\right], \\
\left\{\Phi\left[\chi^{i}\right], \Phi\left[\psi^{j}\right]\right\}= & \Phi\left[\chi^{j} \partial_{j} \psi^{i}-\psi^{j} \partial_{j} \chi^{i}\right], \\
\left\{\mathscr{H}_{T}[\xi], \mathscr{C}\left[\chi^{i}\right]\right\}= & 0, \\
\left\{\Phi\left[\chi^{i}\right], \mathscr{C}\left[\eta^{j}\right]\right\}= & \mathscr{C}\left[\chi^{i} \partial_{j} \eta^{j}\right] .
\end{aligned}
$$

The first three Poisson brackets in (3.39) are the familiar relations found in GR. The constraint in the right-hand side of the first Poisson bracket is just the momentum constraint of GR, $\mathscr{H}_{i}-p_{\lambda} \mathscr{C}_{i}-\partial_{i} V_{\boldsymbol{n}} p_{\boldsymbol{n}}=-2 h_{i j} D_{k} \pi^{j k} \approx 0$. The last two Poisson brackets tell that $\mathscr{C}_{i}$ is preserved in time and that it transforms as a vector density under the spatial diffeomorphisms generated by the momentum constraint. The constraints $\pi_{N}, \pi_{i}, p_{i}$ have vanishing Poisson bracket with every constraint. Thus all the constraints are preserved under time evolution.

We can now see that all the constraints $\left(\mathscr{H}_{T}^{\prime}, \mathscr{H}_{i}, \pi_{N}, \pi_{i}\right.$, $\left.p_{i}, \mathscr{C}_{i}^{\prime}\right)$ in the Hamiltonian (3.36) are first class constraints. The Lagrange multipliers in the Hamiltonian (3.36) remain unspecified, until they are determined as a part of the gauge fixing procedure. In addition, $p_{\boldsymbol{n}} \approx 0$ and $\mathscr{C}_{\lambda} \approx 0$ are the second class constraints.

In order to clarify the nature of the constraint $\mathscr{C}_{i}$, it is useful to decompose the variables $\lambda, p_{\lambda}$ as follows:

$$
\begin{aligned}
\lambda(t, x) & =\lambda_{0}(t)+\bar{\lambda}(t, x), \\
p_{\lambda}(t, x) & =\frac{\sqrt{h}}{\int_{\Sigma_{t}} \mathrm{~d}^{3} x \sqrt{h}} p_{\lambda}^{0}(t)+\bar{p}_{\lambda}(t, x),
\end{aligned}
$$

where the zero modes describe the time-dependent averages of $\lambda$ and $p_{\lambda}$ over space,

$$
\begin{aligned}
& \lambda_{0}(t)=\frac{1}{\int_{\Sigma_{t}} \mathrm{~d}^{3} x \sqrt{h}} \int_{\Sigma_{t}} \mathrm{~d}^{3} x \sqrt{h} \lambda(t, x), \\
& p_{\lambda}^{0}(t)=\int_{\Sigma_{t}} \mathrm{~d}^{3} x p_{\lambda}(t, x),
\end{aligned}
$$

and the barred components have vanishing average values over space,

$$
\int_{\Sigma_{t}} \mathrm{~d}^{3} x \sqrt{h} \bar{\lambda}(t, x)=0, \quad \int_{\Sigma_{t}} \mathrm{~d}^{3} x \bar{p}_{\lambda}(t, x)=0 .
$$

If the space $\Sigma_{t}$ is infinite, the definition of the zero modes (3.41) has to be specified more precisely. For example, in the asymptotically flat case, the spatial integrals would be defined up to a finite radius $r$ in the asymptotic region, and finally the limit $r \rightarrow \infty$ would be taken. In the definition of $\lambda_{0}$ the two infinite integrals cancel out, since the asymptotic value of $\lambda$ must be a constant, so that the average value $\lambda_{0}$ remains finite. The momentum $p_{\lambda}$ can be defined to have such an asymptotic behavior that the definition of its zero mode remains finite. Other scalar fields or scalar densities can be decomposed in a similar way. The zero modes satisfy the canonical Poisson bracket

$$
\left\{\lambda_{0}, p_{\lambda}^{0}\right\}=1
$$

while the average free components satisfy

$$
\left\{\bar{\lambda}(x), \bar{p}_{\lambda}(y)\right\}=\delta(x-y)-\frac{\sqrt{h}(y)}{\int_{\Sigma_{t}} \mathrm{~d}^{3} z \sqrt{h}},
$$

and the Poisson brackets between zero modes and average free components are zero

$$
\left\{\lambda_{0}, \bar{p}_{\lambda}(x)\right\}=0, \quad\left\{\bar{\lambda}(x), p_{\lambda}^{0}\right\}=0 .
$$

When $\lambda$ is decomposed, the constraint (3.30) can be replaced with a local constraint

$\bar{\lambda} \approx 0$,

since $\partial_{i} \lambda=\partial_{i} \bar{\lambda}=0$ implies that $\bar{\lambda}$ is constant over space and the zero average condition (3.42) requires that constant to be zero. The corresponding first class constraint (3.38) is replaced with

$\overline{\mathscr{C}}^{\prime}=\bar{\lambda}-\overline{\left(\frac{p_{\boldsymbol{n}}}{\sqrt{h}}\right)} \approx 0$,

where the overline denotes a component whose integral over space vanishes. The purpose of the decomposition (3.40) of the cosmological variable is to separate the perturbative component $\bar{\lambda}$ that vanishes due to the constraint (3.30). The average component $\lambda_{0}$ is left unconstrained.

The total Hamiltonian (3.36) is rewritten as

$$
\begin{aligned}
H= & \int_{\Sigma_{t}} \mathrm{~d}^{3} x\left(N \mathscr{H}_{T}^{\prime}+N^{i} \mathscr{H}_{i}+v_{N} \pi_{N}+v_{N}^{i} \pi_{i}\right. \\
& \left.+\bar{v}_{\lambda} \overline{\mathscr{C}}^{\prime}+v^{i} p_{i}\right)+H_{\mathscr{B}_{t}},
\end{aligned}
$$

where the variable $\lambda$ in the Hamiltonian constraint (3.37) is replaced with its zero mode $\lambda_{0}$. Next we consider gauge fixing and simplification of the Hamiltonian via elimination of some variables.

\subsubsection{Gauge fixing and the second class constraints}

Each of the first class constraints generates a gauge transformation. The constraint $p_{i}$ generates a gauge transformation of the vector $V^{i}$ as

$\delta V^{i}=\left\{V^{i}, \int_{\Sigma_{t}} \mathrm{~d}^{3} x \varepsilon^{i} p_{i}\right\}=\varepsilon^{i}$,

where $\varepsilon^{i}$ is an infinitesimal gauge parameter. This means that $V^{i}$ can be fixed throughout spacetime as a gauge choice. We 
choose the gauge fixing condition as $V^{i}=0$. We can further simplify the system by considering the gauge symmetry that is associated with the constraint (3.47). The constraint (3.47) generates the transformation of the average free momentum $\bar{p}_{\lambda}$,

$\delta \bar{p}_{\lambda}=\left\{\bar{p}_{\lambda}, \int_{\Sigma_{t}} \mathrm{~d}^{3} x \bar{\varepsilon} \overline{\mathscr{C}}^{\prime}\right\}=-\bar{\varepsilon}$,

where the infinitesimal gauge parameter $\bar{\varepsilon}$ is a now a scalar density whose integral over $\Sigma_{t}$ vanishes. Equivalently, the constraint (3.38) generates the transformation

$\delta \bar{p}_{\lambda}=\left\{\bar{p}_{\lambda}, \int_{\Sigma_{t}} \mathrm{~d}^{3} x \varepsilon^{i} \mathscr{C}_{i}^{\prime}\right\}=\partial_{i} \varepsilon^{i}$,

where the integral of the component of the infinitesimal gauge parameter $\varepsilon^{i}$ in the direction of the outward-pointing unit normal $r_{i}$ to the boundary of $\Sigma_{t}$ is zero, so that $\int_{\Sigma_{t}} \mathrm{~d}^{3} x \partial_{i} \varepsilon^{i}=\int_{\mathscr{B}_{t}} \mathrm{~d}^{2} x r_{i} \varepsilon^{i}=0$. The corresponding gauge freedom can be fixed by setting $\bar{p}_{\lambda}=0$.

Now we have the set of second class constraints $\left(\mathscr{C}_{\lambda}, p_{\boldsymbol{n}}\right.$, $\left.V^{i}, p_{i}, \bar{\lambda}, \bar{p}_{\lambda}\right)$. The second class constraints can be set to zero strongly, if we replace the Poisson bracket with the Dirac bracket. In this case the Dirac bracket is equal to the Poisson bracket. Then we can eliminate six canonical variables $\left(V_{\boldsymbol{n}}, p_{\boldsymbol{n}}, V^{i}, p_{i}, \bar{\lambda}, \bar{p}_{\lambda}\right)$ by using the constraints

$$
\begin{aligned}
V_{\boldsymbol{n}} & =\frac{p_{\lambda}}{\sqrt{h}}, \quad p_{\boldsymbol{n}}=0, \\
V^{i} & =0, \quad p_{i}=0, \\
\bar{\lambda} & =0, \quad \bar{p}_{\lambda}=0 .
\end{aligned}
$$

The Hamiltonian (3.48) is written as

$$
H=\int_{\Sigma_{t}} \mathrm{~d}^{3} x\left(N \mathscr{H}_{T}+N^{i} \mathscr{H}_{i}+v_{N} \pi_{N}+v_{N}^{i} \pi_{i}\right)+H_{\mathscr{B}_{t}},
$$

where

$$
\mathscr{H}_{T}=\frac{\kappa}{\sqrt{h}} \pi^{i j} \mathscr{G}_{i j k l} \pi^{k l}-\frac{\sqrt{h}}{\kappa}^{(3)} R+\sqrt{h} \lambda_{0} \approx 0,
$$

and

$\mathscr{H}_{i}=-2 h_{i j} D_{k} \pi^{j k} \approx 0$.

This is the Hamiltonian of GR with a time-dependent cosmological constant $\lambda_{0}$. However, it is evident that $\lambda_{0}$ is a constant in time as well, since the Hamiltonian is independent of $p_{\lambda}^{0}$,

$\partial_{t} \lambda_{0}=\left\{\lambda_{0}, H\right\}=0$.

The value of $\lambda_{0}$ is set as a part of the initial value data on the initial Cauchy surface, say $\Sigma_{0}$ at $t=0$. The momentum $p_{\lambda}^{0}$ evolves monotonically, $\partial_{t} p_{\lambda}^{0}=-N \sqrt{h}$, and it is not involved in the actual dynamics of the system. The physical degrees of freedom consist of the two standard modes of gravity, plus the nondynamical zero mode that provides the cosmological constant.

Gauge fixing the diffeomorphism invariance is done exactly as in GR by introducing appropriate gauge conditions for the generators $\mathscr{H}_{T}, \mathscr{H}_{i}, \pi_{N}, \pi^{i}$.

\subsection{Path integral}

\subsubsection{Canonical path integral and possible gauges}

The canonical Hamiltonian for the gravitational part of the action (2.13) is written as

$H_{c}=\int_{\Sigma_{t}} \mathrm{~d}^{3} x\left(N \mathscr{H}_{T}+N^{i} \mathscr{H}_{i}\right)+H_{\mathscr{B}_{t}}$,

where the Hamiltonian and momentum constraints are defined in (3.21) and (3.22), and the boundary term in (3.24). The second class constraints are

$p_{\boldsymbol{n}} \approx 0, \quad \mathscr{C}_{\lambda}=p_{\lambda}-\sqrt{h} V_{\boldsymbol{n}} \approx 0$.

The first class constraints are $\pi_{N} \approx 0, \pi_{i} \approx 0, p_{i} \approx 0, \mathscr{H}_{i} \approx$ 0 , and

$$
\begin{aligned}
& \mathscr{H}_{T}^{\prime}=\mathscr{H}_{T}-p_{\boldsymbol{n}}+\frac{\kappa}{2} \frac{h_{i j} \pi^{i j}}{\sqrt{h}} V_{\boldsymbol{n}} p_{\boldsymbol{n}} \approx 0, \\
& \overline{\mathscr{C}}^{\prime}=\bar{\lambda}-\overline{\left(\frac{p_{\boldsymbol{n}}}{\sqrt{h}}\right)} \approx 0 .
\end{aligned}
$$

We introduce gauge fixing conditions as

$\sigma^{0}=N-f \approx 0, \quad \sigma^{i}=N^{i}-f^{i} \approx 0, \quad V^{i} \approx 0$,

$\chi^{\mu}\left(h_{i j}, \pi^{i j}\right) \approx 0, \quad \bar{p}_{\lambda} \approx 0$,

where $f$ and $f^{i}$ are fixed functions (or constants), such that $f>0$, while the conditions $\chi^{\mu}$ can depend on both $h_{i j}$ and $\pi^{i j}$, presuming that $\chi^{\mu}$ depends on $\pi^{i j}$ linearly or not at all. The four gauge conditions $\chi^{\mu}$ have to be independent, so that they fix four components of the variables $h_{i j}, p^{i j}$. Furthermore, it is convenient to require that

$\left\{\chi^{\mu}, \chi^{\nu}\right\}=0$.

The generator $\overline{\mathscr{C}}^{\prime}$ exhibits a nonlocal linear dependence over the spatial hypersurface, since the spatial integral of the generator vanishes by definition. The corresponding gauge condition $\left(\bar{p}_{\lambda} \approx 0\right)$ has a similar nonlocal linear dependence. Quantization of gauge theories with linearly dependent generators [28] is discussed in Appendix A (see also [29]). There we show that the following path integral is obtained when certain additional gauge conditions are imposed on the ghost fields associated with the generator $\overline{\mathscr{C}}^{\prime}$.

Since the first class constraints have vanishing Poisson brackets with every constraint except the gauge conditions, we use the Faddeev formula for the functional determinant of constraints in the path integral. Furthermore, the determinant 
of the Poisson bracket between the gauge conditions and the gauge generators has a block diagonal form, so that it factorizes. Hence we obtain the integration measure as

$$
\begin{aligned}
\prod_{x^{\mu}} & \mathscr{D} N \mathscr{D} \pi_{N} \mathscr{D} N^{i} \mathscr{D} \pi_{i} \mathscr{D} V_{\boldsymbol{n}} \mathscr{D} p_{\boldsymbol{n}} \mathscr{D} V^{i} \mathscr{D} p_{i} \mathscr{D} h_{i j} \mathscr{D} \pi^{i j} \\
& \times \mathscr{D} \lambda_{0} \mathscr{D} p_{\lambda}^{0} \mathscr{D} \bar{\lambda} \mathscr{D} \bar{p}_{\lambda} \delta\left(p_{\boldsymbol{n}}\right) \delta\left(\mathscr{C}_{\lambda}\right) \delta\left(\pi_{N}\right) \delta\left(\pi_{i}\right) \\
& \times \delta\left(\sigma^{\mu}\right) \delta\left(p_{i}\right) \delta\left(V^{i}\right) \delta\left(\mathscr{H}_{\mu}^{\prime}\right) \delta\left(\chi^{\mu}\right) \delta\left(\overline{\mathscr{C}}^{\prime}\right) \delta\left(\bar{p}_{\lambda}\right) \\
& \times \sqrt{h}\left|\operatorname{det}\left\{\chi^{\mu}, \mathscr{H}_{v}^{\prime}\right\}\right|
\end{aligned}
$$

where we denote $\mathscr{H}_{v}^{\prime}=\left(\mathscr{H}_{T}^{\prime}, \mathscr{H}_{i}\right)$. Integration over the variables $N, \pi_{N}, N^{i}, \pi_{i}, V_{\boldsymbol{n}}, p_{\boldsymbol{n}}, V^{i}, p_{i}, \bar{\lambda}, \bar{p}_{\lambda}$ can be performed using the constraint $\delta$-functions. The Hamiltonian and momentum constraints reduce to (3.54) and (3.55), respectively, and we denote them collectively as $\mathscr{H}_{v}=$ $\left(\mathscr{H}_{T}, \mathscr{H}_{i}\right)$. In addition, we write $\delta\left(\mathscr{H}_{\mu}\right)$ as an integral over auxiliary fields $N^{\mu}=\left(N, N^{i}\right)$, essentially reintroducing the lapse and shift functions. The auxiliary fields are displaced so that the gauge fixing functions $\left(f, f^{i}\right)$ are canceled, $N+f \rightarrow$ $N$ and $N^{i}+f^{i} \rightarrow N^{i}$. Then the path integral is written as

$$
\begin{aligned}
Z_{\mathrm{DUG}}= & \mathscr{N}^{-1} \int \prod_{x^{\mu}} \mathscr{D} N \mathscr{D} N^{i} \mathscr{D} h_{i j} \mathscr{D} \pi^{i j} \mathscr{D} \lambda_{0} \mathscr{D} p_{\lambda}^{0} \delta\left(\chi^{\mu}\right) \\
& \times\left|\operatorname{det}\left\{\chi^{\mu}, \mathscr{H}_{\nu}\right\}\right| \exp \left[\frac { i } { \hbar } \int \mathrm { d } t \left(\int _ { \Sigma _ { t } } \left(\pi^{i j} \partial_{t} h_{i j}\right.\right.\right. \\
& \left.\left.\left.+p_{\lambda}^{0} \partial_{t} \lambda_{0}-N \mathscr{H}_{T}-N^{i} \mathscr{H}_{i}\right)-H_{\mathscr{B}_{t}}\right)\right],
\end{aligned}
$$

where $\mathscr{N}$ is a normalization factor. Integration over $p_{\lambda}^{0}$ gives a $\delta$-function that imposes $\partial_{t} \lambda_{0}=0$. Therefore we decompose $\lambda_{0}$ to a constant component and an average free component $\bar{\lambda}_{0}$ over time as

$\lambda_{0}(t)=\frac{2}{\kappa} \Lambda+\bar{\lambda}_{0}(t)$,

where $\int \mathrm{d} t \bar{\lambda}_{0}=0$. The integration over $\bar{\lambda}_{0}$ is performed, which gives

$$
\begin{aligned}
Z_{\mathrm{DUG}}= & \mathscr{N}^{-1} \int \prod_{\chi^{\mu}} \mathscr{D} N \mathscr{D} N^{i} \mathscr{D} h_{i j} \mathscr{D} \pi^{i j} \delta\left(\chi^{\mu}\right) \\
& \times\left|\operatorname{det}\left\{\chi^{\mu}, \mathscr{H}_{v}\right\}\right| \exp \left[\frac { i } { \hbar } \int \mathrm { d } t \left(\int _ { \Sigma _ { t } } \left(\pi^{i j} \partial_{t} h_{i j}\right.\right.\right. \\
& \left.\left.\left.-N \mathscr{H}_{T}-N^{i} \mathscr{H}_{i}\right)-H_{\mathscr{B}_{t}}\right)\right]
\end{aligned}
$$

where we have redefined

$\mathscr{H}_{T}=\frac{\kappa}{\sqrt{h}} \pi^{i j} \mathscr{G}_{i j k l} \pi^{k l}-\frac{\sqrt{h}}{\kappa}{ }^{(3)} R+\frac{2 \sqrt{h}}{\kappa} \Lambda$.

Unlike in [14], the path integral (3.65) does not include integration over the cosmological constant $\Lambda$, since we presume that the boundary conditions of the path integral define the (asymptotic) boundary values of all variables, including the boundary value of $\lambda$. In particular, the value of $\lambda$ is set to a constant both on the initial Cauchy surface and on the spatial boundary. An extension of the path integral with an integral over $\Lambda$ will be considered in Sect. 3.3.2.

Then we perform the integration over the momentum $\pi^{i j}$. Since the measure is at most linear in the momentum, the integration is Gaussian, and hence the integration can be performed in the standard way (see e.g. [30]). The integration amounts to expressing the momentum as

$\pi^{i j}=\frac{\sqrt{h}}{\kappa} \mathscr{G}^{i j k l} K_{k l}$,

and including the factor $N^{-3} h^{-\frac{1}{2}} \cdot 3$ This results in

$$
\begin{aligned}
Z_{\mathrm{DUG}}= & \mathscr{N}^{-1} \int \prod_{x^{\mu}} \mathscr{D} N \mathscr{D} N^{i} \mathscr{D} h_{i j} N^{-3} h^{-\frac{1}{2}} \delta\left(\chi^{\mu}\right) \\
& \times\left|\operatorname{det}\left\{\chi^{\mu}, \mathscr{H}_{\nu}\right\}\right| \exp \left[\frac { i } { \hbar } \left(\frac{1}{\kappa} \int \mathrm{d} t \int_{\Sigma_{t}} N \sqrt{h}\right.\right. \\
& \left.\times\left(K_{i j}\left(\mathscr{G}^{i j k l} K_{k l}+{ }^{(3)} R-2 \Lambda\right)+S_{\mathscr{B}}\right)\right] .
\end{aligned}
$$

Then we express the field differentials as

$\mathscr{D} g_{\mu \nu}=2 h N \mathscr{D} N \mathscr{D} N^{i} \mathscr{D} h_{i j}$,

and write $N^{-4} h^{-\frac{3}{2}}=N g^{00}(-g)^{-\frac{3}{2}}$, and we obtain the path integral as

$$
\begin{aligned}
Z_{\mathrm{DUG}}= & \mathscr{N}^{-1} \int \prod_{x^{\mu}} \mathscr{D} g_{\mu \nu} g^{00}(-g)^{-\frac{3}{2}} N \delta\left(\chi^{\mu}\right) \\
& \times\left|\operatorname{det}\left\{\chi^{\mu}, \mathscr{H}_{\nu}\right\}\right| \exp \left(\frac{i}{\hbar} S_{\mathrm{EH}}\left[g_{\mu \nu}, \Lambda\right]\right),
\end{aligned}
$$

where $S_{\mathrm{EH}}\left[g_{\mu \nu}, \Lambda\right]$ is the Einstein-Hilbert (EH) action with an unspecified cosmological constant $\Lambda$,

$$
\begin{aligned}
S_{\mathrm{EH}}\left[g_{\mu \nu}, \Lambda\right]= & \frac{1}{\kappa} \int_{\mathscr{M}} \mathrm{d}^{4} x \sqrt{-g}(R-2 \Lambda) \\
& +\frac{2}{\kappa} \oint_{\partial \mathscr{M}} \mathrm{d}^{3} x \sqrt{|\gamma|} \mathscr{K} .
\end{aligned}
$$

In summary, the difference compared to GR is that the value of the cosmological constant $\Lambda$ is included in the initial and boundary conditions, rather than being a coupling constant of the Lagrangian.

The next step is to express the gauge fixing factor of (3.70) in a more useful form. For that purpose we consider specific gauge conditions for the Hamiltonian and momentum constraints. The present theory has the advantage of enabling the use of the same gauges for the diffeomorphism symmetry as in GR.

3 The factor comes from $\left|\operatorname{det} \frac{N \kappa}{\sqrt{h}} \mathscr{G}_{i j k l}\right|^{-\frac{1}{2}}=2^{2} \kappa^{-3} N^{-3} h^{-\frac{1}{2}}$, where the de Witt metric $\mathscr{G}_{i j k l}$ is regarded as a symmetric 6 by 6 matrix with indices $(i j)$ and $(k l)$ ranging over the six unique components. 
Dirac gauge First we consider the Dirac gauge [31]:

$\chi_{\mathrm{D}}^{0}=h_{i j} \pi^{i j} \approx 0, \quad \chi_{\mathrm{D}}^{i}=\partial_{j}\left(h^{\frac{1}{3}} h^{i j}\right) \approx 0$.

We define an operator $Q_{\mathrm{D} v}^{\mu}$ in terms of the gauge transformation of the gauge conditions (3.72) as

$Q_{\mathrm{D} \nu}^{\mu} \xi^{\nu}=\left\{\chi_{\mathrm{D}}^{\mu}, \int_{\Sigma_{t}} \mathscr{H}_{\nu} \xi^{\nu}\right\}, \quad \xi^{\mu}=\left(\xi, \xi^{i}\right)$.

Evaluating the Poisson brackets, we obtain the components of the operator as (up to the Hamiltonian constraint)

$$
\begin{aligned}
Q_{\mathrm{D} 0}^{0}= & -\frac{2}{\kappa} \sqrt{h}\left(h^{i j} D_{i} D_{j}-{ }^{(3)} R+3 \Lambda\right), \\
Q_{\mathrm{D} i}^{0}= & -\chi_{\mathrm{D}}^{0} \partial_{i}-\partial_{i} \chi_{\mathrm{D}}^{0}, \\
Q_{\mathrm{D} 0}^{i}= & -2 h^{\frac{1}{3}}\left(K^{i j}-\frac{1}{3} h^{i j} K\right) \partial_{j} \\
& -2 \partial_{j}\left[h^{\frac{1}{3}}\left(K^{i j}-\frac{1}{3} h^{i j} K\right)\right], \\
Q_{\mathrm{D} j}^{i}= & -h^{\frac{1}{3}}\left(\delta^{i}{ }_{j} h^{k l} \partial_{k} \partial_{l}+\frac{1}{3} h^{i k} \partial_{k} \partial_{j}\right)-\delta_{j}^{i} \chi_{\mathrm{D}}^{k} \partial_{k} \\
& +\frac{2}{3} \chi_{\mathrm{D}}^{i} \partial_{j}+\partial_{j} \chi_{\mathrm{D}}^{i},
\end{aligned}
$$

where the momentum $\pi^{i j}$ is written in terms of the metric (3.67) and we denote $K^{i j}=h^{i k} h^{j l} K_{k l}$. In order to obtain a gauge-invariant form for the functional determinant [32], an extra factor $N$ is included into the components $Q_{\mathrm{D} \mu}^{0}$. Hence we replace (3.74) with

$Q_{\mathrm{D} 0}^{0}=-\frac{2}{\kappa} \sqrt{-g}\left(h^{i j} D_{i} D_{j}-{ }^{(3)} R+3 \Lambda\right)$,

and (3.75) with $Q_{\mathrm{D} i}^{0}=-N \chi_{\mathrm{D}}^{0} \partial_{i}-N \partial_{i} \chi_{\mathrm{D}}^{0}$. For practical applications, the components of the operator $Q_{\mathrm{D} v}^{\mu}$ could be simplified by using the constraints, in particular the gauge conditions (3.72), and even further using the quasiclassical approximation (see [32]).

Finally, the path integral can be written as

$$
\begin{aligned}
& Z_{\mathrm{DUG}}=\mathscr{N}^{-1} \int \prod_{x^{\mu}} \mathscr{D} g_{\mu \nu} \mathscr{D} \eta_{\rho} \mathscr{D} c^{*} \mathscr{D} c \mathscr{D} c_{i}^{*} \mathscr{D} c^{i} g^{00}(-g)^{-\frac{3}{2}} \\
& \quad \times \exp \left[\frac { i } { \hbar } \left(S_{\mathrm{EH}}\left[g_{\mu \nu}, \Lambda\right]-\int_{\mathscr{M}} \mathrm{d}^{4} x\left(\eta_{\mu} \chi_{\mathrm{D}}^{\mu}+c^{*} Q_{\mathrm{D} 0}^{0} c\right.\right.\right. \\
& \left.\left.\left.+c^{*} Q_{\mathrm{D} i}^{0} c^{i}+c_{i}^{*} Q_{\mathrm{D} 0}^{i} c+c_{i}^{*} Q_{\mathrm{D} j}^{i} c^{j}\right)\right)\right]
\end{aligned}
$$

where we have introduced pairs of anti-commuting fields $c, c^{*}$, and $c^{i}, c_{i}^{*}$, commonly referred to as Faddeev-Popov ghosts (and anti-ghosts), and an auxiliary field $\eta_{\mu}$ for each gauge condition $\chi_{\mathrm{D}}^{\mu}$. Evidently, the full expression for the action is noncovariant in the Dirac gauge.

Transverse harmonic gauge:

$\chi^{\mu}=\partial_{\nu} \hat{g}^{\mu \nu} \approx 0 ; \quad \hat{g}^{\mu \nu}=\sqrt{-g} g^{\mu \nu}$.
Transforming to this covariant gauge is achieved via the Faddeev-Popov trick in the same way as in GR. The operator corresponding to this gauge is again obtained from the gauge transformation of the gauge conditions (3.80) as

$Q_{\nu}^{\mu} \xi^{\nu}=\delta_{\xi} \chi^{\mu}=\partial_{\nu}\left(\partial_{\rho}\left(\hat{g}^{\mu \nu} \xi^{\rho}\right)-\hat{g}^{\mu \rho} \partial_{\rho} \xi^{\nu}-\hat{g}^{\rho \nu} \partial_{\rho} \xi^{\mu}\right)$.

Thanks to the gauge-invariant form of the integration measure [32], the path integral is obtained as

$$
\begin{aligned}
& Z_{\mathrm{DUG}}=\mathscr{N}^{-1} \int \prod_{x^{\mu}} \mathscr{D} g_{\mu \nu} \mathscr{D} \eta_{\rho} \mathscr{D} c_{\sigma}^{*} \mathscr{D} c^{\sigma} g^{00}(-g)^{-\frac{3}{2}} \\
& \times \exp \left[\frac { i } { \hbar } \left(S_{\mathrm{EH}}\left[g_{\mu \nu}, \Lambda\right]+\int_{\mathscr{M}} \mathrm{d}^{4} x\left(-\eta_{\mu} \chi^{\mu}+\partial_{\mu} c_{v}^{*}\right.\right.\right. \\
& \left.\left.\left.\quad \times\left(\partial_{\rho}\left(\hat{g}^{\mu \nu} c^{\rho}\right)-\hat{g}^{\mu \rho} \partial_{\rho} c^{v}-\hat{g}^{\rho \nu} \partial_{\rho} c^{\mu}\right)\right)\right)\right]
\end{aligned}
$$

where $c^{\mu}$ and $c_{\mu}^{*}$ are the Faddeev-Popov ghosts.

Matter can be included similarly as in GR. For simplicity we assume that no extra gauge symmetries or constraints are involved. Finally, we define the generating functional by including external source $J^{\mu \nu}$ and $J_{\Psi}$ for the metric and the matter fields $\Psi$, respectively,

$$
\begin{aligned}
& Z_{\mathrm{DUG}}[J]=\int \prod_{x^{\mu}} \mathscr{D} g_{\mu \nu} \mathscr{D} \eta_{\rho} \mathscr{D} c_{\sigma}^{*} \mathscr{D} c^{\sigma} \mathscr{D} \Psi g^{00}(-g)^{-\frac{3}{2}} \\
& \quad \times \exp \left[\frac { i } { \hbar } \left(S_{\mathrm{EH}}\left[g_{\mu \nu}, \Lambda\right]+S_{\mathrm{m}}\left[g_{\mu \nu}, \Psi\right]+\int_{\mathscr{M}} \mathrm{d}^{4} x\left(-\eta_{\mu} \chi^{\mu}\right.\right.\right. \\
& \quad+\partial_{\mu} c_{\nu}^{*}\left(\partial_{\rho}\left(\hat{g}^{\mu \nu} c^{\rho}\right)-\hat{g}^{\mu \rho} \partial_{\rho} c^{\nu}-\hat{g}^{\rho \nu} \partial_{\rho} c^{\mu}\right) \\
& \left.\left.\left.\quad+g_{\mu \nu} J^{\mu \nu}+\Psi J_{\Psi}\right)\right)\right] .
\end{aligned}
$$

We have shown that the path integral for the fully diffeomorphism-invariant unimodular gravity (2.13) has the same form as the path integral for GR with a cosmological constant. The crucial difference from GR is that the value of the cosmological constant is set as a part of the boundary conditions for the path integral.

The quantum effective action for the DUG theory can be defined in the exact same way as for GR, since there are no extra conditions on the metric and the path integrals have the same form.

\subsection{2 $\mathrm{Ng}$ and van Dam form of the path integral}

The path integral for unimodular gravity can be extended by including an integration over the cosmological constant $\Lambda$. Then the path integral takes the following form:

$Z_{\mathrm{NvD}}=\int \mathrm{d} \mu(\Lambda) Z_{\mathrm{DUG}}(\Lambda)$

where $\mathrm{d} \mu(\Lambda)$ is an integration measure for $\Lambda$, and the path integral for unimodular gravity, $Z_{\mathrm{DUG}}(\Lambda)$, is given in (3.82) 
with boundary conditions chosen to be consistent with a given value $\Lambda$ of the cosmological constant. This form of the path integral for unimodular gravity was originally proposed in $[12,13]$. It was also later derived from a canonical path integral [14], although some manipulation of variables was required, and the canonical measure was assumed to include an integral over $\Lambda$. In [14], the integral over $\lambda_{0}$ was assumed to include integration over both $\Lambda$ and $\bar{\lambda}_{0}$ due to the decomposition (3.64). Here we show that the path integral (3.84) follows straightforwardly from the canonical path integral of the action (2.13), when we consider the vacuum state of the universe to be a superposition of the states corresponding to different values of $\Lambda$ [5].

We emphasize that (3.84) is a quite different path integral compared to the one we derived above (3.82). In the path integral (3.82), the value of the cosmological constant is set as a part of the physical boundary conditions, which (together with a semiclassical matter distribution) define the vacuum state of the system. Including an additional integration over $\Lambda$ means that we are integrating over different boundary conditions, i.e., vacuums. Below we attempt to justify the integration of $\Lambda$ properly.

Let $|\Lambda\rangle$ denote the vacuum state of the universe that is consistent with a given value of the cosmological constant $\Lambda$ and with other relevant boundary conditions. The path integral that we have obtained for the fully diffeomorphisminvariant unimodular gravity (3.82) represents the vacuum transition amplitude

$\langle\Lambda \mid \Lambda\rangle=Z_{\mathrm{DUG}}(\Lambda)$.

We assume that transitions between vacuums are prohibited if the vacuums correspond to different values of $\Lambda$, i.e., the states $|\Lambda\rangle$ are assumed to be orthogonal,

$\left\langle\Lambda \mid \Lambda^{\prime}\right\rangle=0$ if $\Lambda \neq \Lambda^{\prime}$.

Furthermore we assume that the states are nondegenerate, i.e., there exists one state $|\Lambda\rangle$ for each value of $\Lambda$. The vacuum state of the universe is written as a superposition of the states corresponding to different values of $\Lambda$ as

$|\Omega\rangle=\int \mathrm{d} \Lambda \omega(\Lambda)|\Lambda\rangle$.

Choosing the weight function $\omega(\Lambda)$ defines which states $|\Lambda\rangle$ are included in the superposition. Now the vacuum transition amplitude is obtained in the form (3.84) as

$\langle\Omega \mid \Omega\rangle=\int \mathrm{d} \Lambda|\omega(\Lambda)|^{2}\langle\Lambda \mid \Lambda\rangle=\int \mathrm{d} \mu(\Lambda) Z_{\mathrm{DUG}}(\Lambda)$,

where the measure $\mathrm{d} \mu(\Lambda)$ is defined by the weight function as

$\mathrm{d} \mu(\Lambda)=|\omega(\Lambda)|^{2} \mathrm{~d} \Lambda$.
Using the semiclassical approximation and then the stationary phase approximation, it was argued in [12] that the path integral (3.84) for pure gravity is dominated by solutions whose cosmological constant $\Lambda=0$. In the presence of matter (3.83), the same argument was used in [14] to see that the path integral (3.84) is dominated by the solutions of the Einstein equation whose cosmological constant is approximately

$\Lambda=2 \pi G \frac{\int_{\mathscr{M}} \sqrt{-g} \rho}{\int_{\mathscr{M}} \sqrt{-g}}$,

where $\rho$ is the energy density of a perfect fluid. This result was argued to imply that (3.90) is the most likely value of the cosmological constant. It is intriguing that using the present day energy density as an estimate for the average density [14], one obtains a result that is surprisingly close to the observed value of $\Lambda$ (the observed $\Lambda / G$ being about three times the present average energy density).

The result (3.90) is based on a hidden assumption that the given value of $\Lambda$ is included in the vacuum state (3.87). It was assumed that all states $|\Lambda\rangle$ are weighted equally, $|\omega(\Lambda)|^{2}=$ constant. This corresponds to a total lack of physical boundary conditions regarding $\Lambda$, and then using the path integral for finding the most likely value of $\Lambda$. This is an interesting argument, but speculative and conceptually problematic. We indeed need information on the boundary conditions in order to estimate the average value of $\rho$ over spacetime. Even if we accept (3.90) as a valid estimate for the value of $\Lambda$ in our universe, estimating the average of matter energy density over the whole spacetime is challenging, to say the least.

\section{Quantization of the unimodular gravity with a fixed metric determinant}

\subsection{ADM decomposition of the action}

The gravitational part of the action (2.6) is written in ADM form as

$$
\begin{gathered}
S_{\mathrm{UG}}\left[N, N^{i}, h_{i j}, \lambda, \Psi\right]=\int \mathrm{d} t \int_{\Sigma_{t}} \mathrm{~d}^{3} x\left[\frac { N \sqrt { h } } { \kappa } \left(K_{i j} \mathscr{G}^{i j k l} K_{k l}\right.\right. \\
\left.\left.+{ }^{(3)} R\right)-\lambda\left(N \sqrt{h}-\varepsilon_{0}\right)\right]+S_{\mathscr{B}}+S_{\mathrm{m}}\left[g_{\mu \nu}, \Psi\right] .
\end{gathered}
$$

A Hamiltonian formulation of an action of this form has been considered in [27], and our following analysis is similar in several ways.

\subsection{Hamiltonian analysis}

The momenta conjugate to $N, N^{i}$, and $\lambda$ are the primary constraints: 
$\pi_{N} \approx 0, \quad \pi_{i} \approx 0, \quad p_{\lambda} \approx 0$

The Hamiltonian is obtained as

$$
\begin{aligned}
H= & \int_{\Sigma_{t}} \mathrm{~d}^{3} x\left(N \mathscr{H}_{T}+N^{i} \mathscr{H}_{i}-\varepsilon_{0} \lambda+v_{N} \pi_{N}\right. \\
& \left.+v_{N}^{i} \pi_{i}+v_{\lambda} p_{\lambda}\right)+H_{\mathscr{B}_{t}},
\end{aligned}
$$

where the super-Hamiltonian is defined as

$\mathscr{H}_{T}=\frac{\kappa}{\sqrt{h}} \pi^{i j} \mathscr{G}_{i j k l} \pi^{k l}-\frac{\sqrt{h}}{\kappa}^{(3)} R+\sqrt{h} \lambda$,

the supermomentum is defined as

$\mathscr{H}_{i}=-2 h_{i j} D_{k} \pi^{j k}$

$v_{N}, v_{N}^{i}, v_{\lambda}$ are Lagrange multipliers, and $\varepsilon_{0}$ is the fixed scalar density.

Preservation of the primary constraints implies the secondary constraints:

$\mathscr{H}_{T} \approx 0, \quad \mathscr{H}_{i} \approx 0, \quad \mathscr{U}=N \sqrt{h}-\varepsilon_{0} \approx 0$.

The momentum constraint (4.5) can again be extended with terms that are proportional to the primary constraints $\pi_{N}$ and $p_{\lambda}$,

$\mathscr{H}_{i}=-2 h_{i j} D_{k} \pi^{j k}+\partial_{i} N \pi_{N}+\partial_{i} \lambda p_{\lambda}$,

since then it will generate spatial diffeomorphisms on $\Sigma_{t}$ for all the variables that are involved in the secondary constraints (4.6). The smeared Hamiltonian and momentum constraints (3.28) satisfy the following Poisson brackets:

$$
\begin{aligned}
\left\{\mathscr{H}_{T}[\xi], \mathscr{H}_{T}[\eta]\right\}= & \int_{\Sigma_{t}} \mathrm{~d}^{3} x\left(\xi \partial_{i} \eta-\eta \partial_{i} \xi\right) h^{i j} \\
& \times\left(\mathscr{H}_{j}-\partial_{j} N \pi_{N}-\partial_{j} \lambda p_{\lambda}\right), \\
\left\{\Phi\left[\chi^{i}\right], \mathscr{H}_{T}[\xi]\right\}= & \mathscr{H}_{T}\left[\chi^{i} \partial_{i} \xi\right], \\
\left\{\Phi\left[\chi^{i}\right], \Phi\left[\psi^{j}\right]\right\}= & \Phi\left[\chi^{j} \partial_{j} \psi^{i}-\psi^{j} \partial_{j} \chi^{i}\right] .
\end{aligned}
$$

The Hamiltonian and momentum constraints have nonvanishing Poisson brackets with $\mathscr{U}$ :

$$
\begin{aligned}
& \left\{\mathscr{U}, \mathscr{H}_{T}[\xi]\right\}=-\frac{\kappa}{2} N \xi h_{i j} \pi^{i j} \approx-\frac{\kappa}{2} \varepsilon_{0} \xi \frac{h_{i j} \pi^{i j}}{\sqrt{h}}, \\
& \left\{\mathscr{U}, \Phi\left[\chi^{i}\right]\right\}=\chi^{i} \partial_{i}(N \sqrt{h})+\partial_{i} \chi^{i} N \sqrt{h} \approx \varepsilon_{0} \partial_{i} \chi^{i} .
\end{aligned}
$$

Hence the preservation of $\mathscr{U}$,

$$
\begin{aligned}
\partial_{t} \mathscr{U}= & \{\mathscr{U}, H\} \approx-N \frac{\kappa \varepsilon_{0}}{2 \sqrt{h}} h_{i j} \pi^{i j} \\
& +\varepsilon_{0} \partial_{i} N^{i}+\sqrt{h} v_{N} \approx 0,
\end{aligned}
$$

is ensured by fixing the Lagrange multiplier $v_{N}$ as

$$
v_{N}=w_{N} \equiv N \frac{\kappa \varepsilon_{0}}{2 h} h_{i j} \pi^{i j}-\frac{\varepsilon_{0}}{\sqrt{h}} \partial_{i} N^{i} .
$$

The preservation of $\mathscr{H}_{T}$,

$\partial_{t} \mathscr{H}_{T}=\left\{\mathscr{H}_{T}, H\right\} \approx \sqrt{h} v_{\lambda} \approx 0$,

fixes the Lagrange multiplier $v_{\lambda}$ as

$v_{\lambda}=0$.

The preservation of $\mathscr{H}_{i} \approx 0$,

$\partial_{t} \mathscr{H}_{i}=\left\{\mathscr{H}_{i}, H\right\} \approx \varepsilon_{0} \partial_{i} \lambda \approx 0$,

requires the introduction of the secondary constraint (3.30), which was also present in the generally covariant formulation. The constraint (3.30) is preserved in time since the Lagrange multiplier of the primary constraint $p_{\lambda} \approx 0$ has been fixed to zero (4.14). We do not need any further constraints, but we still need to analyze and classify the existing constraints properly.

We again decompose the variables $\lambda, p_{\lambda}$ as in (3.40) and replace the constraint (3.30) with (3.46). The second class constraints $\bar{\lambda} \approx 0, \bar{p}_{\lambda} \approx 0$ can be used to eliminate the average free variables $\bar{\lambda}, \bar{p}_{\lambda}$. Since the Hamiltonian constraint $\mathscr{H}_{T}$ contains the remaining zero mode $\lambda_{0}$, and the zero mode $p_{\lambda}^{0}$ of the primary constraint $p_{\lambda} \approx 0$ remains, we should also decompose $\mathscr{H}_{T}$ as

$$
\begin{aligned}
\mathscr{H}_{T} & =\frac{\sqrt{h}}{\int_{\Sigma_{t}} \mathrm{~d}^{3} x \sqrt{h}} \mathscr{H}_{0}+\overline{\mathscr{H}}_{T}, \\
\mathscr{H}_{0} & =\int_{\Sigma_{t}} \mathrm{~d}^{3} x \mathscr{H}_{T}, \quad \int_{\Sigma_{t}} \mathrm{~d}^{3} x \overline{\mathscr{H}}_{T}=0,
\end{aligned}
$$

where the zero mode and the average free component are, respectively, defined as

$$
\begin{aligned}
\mathscr{H}_{0}= & \int_{\Sigma_{t}} \mathrm{~d}^{3} x\left(\frac{\kappa}{\sqrt{h}} \pi^{i j} \mathscr{G}_{i j k l} \pi^{k l}-\frac{\sqrt{h}}{\kappa}{ }^{(3)} R\right) \\
& +\lambda_{0} \int_{\Sigma_{t}} \mathrm{~d}^{3} x \sqrt{h} \approx 0
\end{aligned}
$$

and

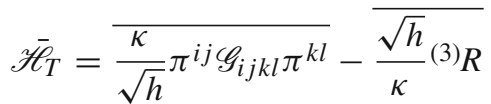

$$
\begin{aligned}
& =\frac{\kappa}{\sqrt{h}} \pi^{i j} \mathscr{G}_{i j k l} \pi^{k l}-\frac{\sqrt{h}}{\kappa}^{(3)} R-\frac{\sqrt{h}}{\int_{\Sigma_{t}} \mathrm{~d}^{3} x \sqrt{h}} \\
& \times \int_{\Sigma_{t}} \mathrm{~d}^{3} x\left(\frac{\kappa}{\sqrt{h}} \pi^{i j} \mathscr{G}_{i j k l} \pi^{k l}-\frac{\sqrt{h}}{\kappa}{ }^{(3)} R\right) \approx 0 .
\end{aligned}
$$


Note that (4.18) does not involve the cosmological variable $\lambda_{0}$. The components $\mathscr{H}_{0}$ and $\overline{\mathscr{H}}_{T}$ satisfy the Poisson brackets

$$
\begin{aligned}
\left\{\mathscr{H}_{0}, \mathscr{H}_{0}\right\}= & 0, \\
\left\{\mathscr{H}_{0}, \overline{\mathscr{H}}_{T}[\bar{\xi}]\right\}= & \int_{\Sigma_{t}} \mathrm{~d}^{3} x \partial_{i} \bar{\xi} h^{i j}\left(\mathscr{H}_{j}-\partial_{j} N \pi_{N}-\partial_{j} \lambda p_{\lambda}\right), \\
\left\{\overline{\mathscr{H}}_{T}[\bar{\xi}], \overline{\mathscr{H}}_{T}[\bar{\eta}]\right\}= & \int_{\Sigma_{t}} \mathrm{~d}^{3} x\left(\bar{\xi} \partial_{i} \bar{\eta}-\bar{\eta} \partial_{i} \bar{\xi}\right) h^{i j} \\
& \times\left(\mathscr{H}_{j}-\partial_{j} N \pi_{N}-\partial_{j} \lambda p_{\lambda}\right),
\end{aligned}
$$

where the smeared constraint $\overline{\mathscr{H}}_{T}[\bar{\xi}]$ is defined so that

$\mathscr{H}_{T}[\xi]=\xi_{0} \mathscr{H}_{0}+\overline{\mathscr{H}}_{T}[\bar{\xi}], \quad \overline{\mathscr{H}}_{T}[\bar{\xi}]=\int_{\Sigma_{t}} \mathrm{~d}^{3} x \bar{\xi}_{\overline{\mathscr{H}}}$,

and where $\xi$ is decomposed as any scalar, $\xi=\xi_{0}+\bar{\xi}$, and

$\xi_{0}=\frac{1}{\int_{\Sigma_{t}} \mathrm{~d}^{3} x \sqrt{h}} \int_{\Sigma_{t}} \mathrm{~d}^{3} x \sqrt{h} \xi, \quad \int_{\Sigma_{t}} \mathrm{~d}^{3} x \sqrt{h} \bar{\xi}=0$.

In the Hamiltonian (4.3), we obtain

$$
\begin{gathered}
\int_{\Sigma_{t}} \mathrm{~d}^{3} x\left(N \mathscr{H}_{T}+N^{i} \mathscr{H}_{i}+w_{N} \pi_{N}\right) \\
=\int_{\Sigma_{t}} \mathrm{~d}^{3} x\left(N \mathscr{H}_{T}^{\prime}+N^{i} \mathscr{H}_{i}^{\prime}\right),
\end{gathered}
$$

where we have extended the Hamiltonian and momentum constraints as

$$
\begin{aligned}
& \mathscr{H}_{T}^{\prime}=\mathscr{H}_{T}+\frac{\kappa}{2} \varepsilon_{0} \frac{h_{i j} \pi^{i j}}{\sqrt{h}} \frac{\pi_{N}}{\sqrt{h}} \approx 0, \\
& \mathscr{H}_{i}^{\prime}=\mathscr{H}_{i}+\varepsilon_{0} \partial_{i}\left(\frac{\pi_{N}}{\sqrt{h}}\right) \approx 0,
\end{aligned}
$$

and furthermore

$$
\int_{\Sigma_{t}} \mathrm{~d}^{3} x N \mathscr{H}_{T}^{\prime}=N_{0} \mathscr{H}_{0}^{\prime}+\int_{\Sigma_{t}} \mathrm{~d}^{3} x \bar{N} \overline{\mathscr{H}}_{T}^{\prime}
$$

where the zero mode and average free component of $\mathscr{H}_{T}^{\prime}$ are defined in the same way as for $\mathscr{H}_{T}$ in (4.16)-(4.18). The first class constraints are the average free Hamiltonian constraint $\overline{\mathscr{H}}_{T}^{\prime}$ and the constraints $\mathscr{H}_{i}^{\prime}, \pi_{i}$. These constraints are associated with the invariance of the action (2.6) under the metric determinant-preserving diffeomorphism (2.1)-(2.5). Since the lapse $N$ is not an unspecified multiplier in the Hamiltonian, we should add the term $\bar{v}_{T} \overline{\mathscr{H}}_{T}^{\prime}$ into the Hamiltonian density, where $\bar{v}_{T}$ is an unspecified Lagrange multiplier. The Hamiltonian (4.3) is rewritten as

$$
\begin{aligned}
H= & \int_{\Sigma_{t}} \mathrm{~d}^{3} x\left(N \mathscr{H}_{T}^{\prime}-\varepsilon_{0} \lambda_{0}+\bar{v}_{T} \overline{\mathscr{H}}_{T}^{\prime}+N^{i} \mathscr{H}_{i}^{\prime}+v_{N}^{i} \pi_{i}\right) \\
& +H_{\mathscr{B}_{t}} .
\end{aligned}
$$

The local constraints $\pi_{N} \approx 0, \mathscr{U} \approx 0$, and the zero mode constraints $p_{\lambda}^{0} \approx 0, \mathscr{H}_{0} \approx 0$ are the second class constraints. The second class constraints $\pi_{N} \approx 0, \mathscr{U} \approx 0$ can be used to eliminate the variables $N, \pi_{N}$ as
$N=\frac{\varepsilon_{0}}{\sqrt{h}}, \quad \pi_{N}=0$.

The zero mode constraints $p_{\lambda}^{0} \approx 0, \mathscr{H}_{0} \approx 0$ can be used to eliminate the variables $\lambda_{0}, p_{\lambda}^{0}$ as

$$
\begin{aligned}
& \lambda_{0}=-\frac{1}{\int_{\Sigma_{t}} \mathrm{~d}^{3} x \sqrt{h}} \int_{\Sigma_{t}} \mathrm{~d}^{3} x\left(\frac{\kappa}{\sqrt{h}} \pi^{i j} \mathscr{G}_{i j k l} \pi^{k l}-\frac{\sqrt{h}}{\kappa}\left({ }^{3)} R\right),\right. \\
& p_{\lambda}^{0}=0 .
\end{aligned}
$$

The Dirac bracket that corresponds to the second class constraints $\left(\pi_{N} \approx 0, \mathscr{U} \approx 0, p_{\lambda}^{0} \approx 0, \mathscr{H}_{0} \approx 0\right.$ ) can be shown to be equal to Poisson bracket for all the remaining variables.

When the second class constraints are set to zero strongly and the auxiliary variables are eliminated as (4.26) and (4.27), we obtain the Hamiltonian as

$$
\begin{aligned}
H= & \frac{\int_{\Sigma_{t}} \mathrm{~d}^{3} x \varepsilon_{0}}{\int_{\Sigma_{t}} \mathrm{~d}^{3} x \sqrt{h}} \int_{\Sigma_{t}} \mathrm{~d}^{3} x\left(\frac{\kappa}{\sqrt{h}} \pi^{i j} \mathscr{G}_{i j k l} \pi^{k l}-\frac{\sqrt{h}}{\kappa}{ }^{(3)} R\right) \\
& +\int_{\Sigma_{t}} \mathrm{~d}^{3} x\left[\left(\frac{\varepsilon_{0}}{\sqrt{h}}+\bar{v}_{T}\right) \overline{\mathscr{H}}_{T}+N^{i} \mathscr{H}_{i}+v_{N}^{i} \pi_{i}\right] \\
& +H_{\mathscr{B}_{t}} .
\end{aligned}
$$

\subsection{Path integral}

The canonical Hamiltonian for the action (2.6) is written as

$H_{c}=\int_{\Sigma_{t}} \mathrm{~d}^{3} x\left(N \mathscr{H}_{T}+N^{i} \mathscr{H}_{i}-\varepsilon_{0} \lambda\right)+H_{\mathscr{B}_{t}}$,

where the Hamiltonian and momentum constraints are defined in (4.4) and (4.5). The second class constraints are

$$
\begin{aligned}
\mathscr{U}=N \sqrt{h}-\varepsilon_{0} & \approx 0, \quad \pi_{N} \approx 0, \\
\bar{\lambda} & \approx 0, \quad \bar{p}_{\lambda} \approx 0, \\
\mathscr{H}_{0} & \approx 0, \quad p_{\lambda}^{0} \approx 0 .
\end{aligned}
$$

The first class constraints are $\pi_{i} \approx 0$ and

$\overline{\mathscr{H}}_{T}^{\prime}=\overline{\mathscr{H}}_{T}+\bar{\kappa} \frac{\kappa}{2} \varepsilon_{0} \frac{h_{i j} \pi^{i j}}{\sqrt{h}} \frac{\pi_{N}}{\sqrt{h}} \approx 0$,

$\mathscr{H}_{i}^{\prime}=\mathscr{H}_{i}+\varepsilon_{0} \partial_{i}\left(\frac{\pi_{N}}{\sqrt{h}}\right) \approx 0$.

We denote the latter two constraints collectively as $\tilde{\mathscr{H}}_{\mu}^{\prime}=$ $\left(\overline{\mathscr{H}}_{T}^{\prime}, \mathscr{H}_{i}^{\prime}\right)$.

The gauge fixing condition for $N^{i}$ is defined as in (3.60), but there is no gauge condition for $N$ due to the first pair of second class constraints in (4.30). The gauge conditions read

$\sigma^{i}=N^{i}-f^{i} \approx 0, \quad \tilde{\chi}^{\mu}\left[h_{i j}, \pi^{i j}\right] \approx 0$,

where one of the conditions $\tilde{\chi}^{\mu}$ has to be average free, so that the number of gauge conditions matches the number of generators exactly. We choose it to be the zero-component, since the zero mode of the super-Hamiltonian is a second class constraint, and hence we denote $\tilde{\chi}^{\mu}=\left(\bar{\chi}^{0}, \chi^{i}\right)$. 
The generator $\overline{\mathscr{H}}_{T}^{\prime}$ and the gauge condition $\bar{\chi}^{0}$ both suffer from a nonlocal linear dependence over the spatial hypersurface, since their spatial integrals vanish by definition. The proper treatment of linearly dependent generators [28] is discussed in Appendix A.

The canonical integration measure for the path integral is written as

$$
\begin{aligned}
\prod_{x^{\mu}} & \mathscr{D} N \mathscr{D} \pi_{N} \mathscr{D} N^{i} \mathscr{D} \pi_{i} \mathscr{D} h_{i j} \mathscr{D} \pi^{i j} \mathscr{D} \lambda_{0} \mathscr{D} p_{\lambda}^{0} \mathscr{D} \bar{\lambda} \mathscr{D} \bar{p}_{\lambda} \delta(\mathscr{U}) \\
& \times \delta\left(\pi_{N}\right) \delta(\bar{\lambda}) \delta\left(\bar{p}_{\lambda}\right) \delta\left(\pi_{i}\right) \delta\left(\sigma^{j}\right) \delta(\mathscr{H} 0) \delta\left(p_{\lambda}^{0}\right) \delta\left(\tilde{\mathscr{H}}_{\mu}^{\prime}\right) \delta\left(\tilde{\chi}^{\mu}\right) \\
& \times\left(\sqrt{h} \int_{\Sigma_{t}} \sqrt{h}\right)\left|\operatorname{det}\left\{\tilde{\chi}^{\mu}, \tilde{\mathscr{H}}_{v}^{\prime}\right\}\right| .
\end{aligned}
$$

The initial and boundary conditions on the cosmological variable are similar to DUG, i.e., the value of $\lambda$ is set to a constant on the initial Cauchy surface and on the spatial boundary. When integration over the variables $N, \pi_{N}, N^{i}, \pi_{i}, \bar{\lambda}, \bar{p}_{\lambda}$, and $p_{\lambda}^{0}$ is performed using the constraints, we obtain

$$
\begin{aligned}
Z_{\mathrm{UG}}= & \mathscr{N}^{-1} \int \prod_{x^{\mu}} \mathscr{D} h_{i j} \mathscr{D} \pi^{i j} \mathscr{D} \lambda_{0} \delta\left(\mathscr{H}_{0}\right) \delta\left(\tilde{\mathscr{H}}_{\mu}\right) \delta\left(\tilde{\chi}^{\mu}\right) \\
& \times\left(\int_{\Sigma_{t}} \sqrt{h}\right)\left|\operatorname{det}\left\{\tilde{\chi}^{\mu}, \tilde{\mathscr{H}}_{v}\right\}\right| \\
& \times \exp \left[\frac { i } { \hbar } \int \mathrm { d } t \left(\int _ { \Sigma _ { t } } \left(\pi^{i j} \partial_{t} h_{i j}-\frac{\varepsilon_{0}}{\sqrt{h}} \mathscr{H}_{T}\right.\right.\right. \\
& \left.\left.\left.-f^{i} \mathscr{H}_{i}+\varepsilon_{0} \lambda_{0}\right)-H_{\mathscr{B}_{t}}\right)\right]
\end{aligned}
$$

where we denote $\tilde{\mathscr{H}}_{v}=\left(\overline{\mathscr{H}}_{T}, \mathscr{H}_{i}\right)$ and the Hamiltonian constraint is given in (3.54). Expressing the $\delta$-functions $\delta\left(\mathscr{H}_{0}\right)$ and $\delta\left(\tilde{\mathscr{H}}_{\mu}\right)$ in terms of integrals over the auxiliary variables $N=\left(N_{0}, \bar{N}\right)$ and $N^{i}$, and shifting the variables as $N \rightarrow N-\frac{\varepsilon_{0}}{\sqrt{h}}$ and $N^{i} \rightarrow N^{i}-f^{i},{ }^{4}$ we obtain

$$
\begin{aligned}
Z_{\mathrm{UG}}= & \mathscr{N}^{-1} \int \prod_{x^{\mu}} \mathscr{D} N \mathscr{D} N^{i} \mathscr{D} h_{i j} \mathscr{D} \pi^{i j} \mathscr{D} \lambda_{0}\left(\int_{\Sigma_{t}} \sqrt{h}\right) \\
& \times \delta\left(\tilde{\chi}^{\mu}\right)\left|\operatorname{det}\left\{\tilde{\chi}^{\mu}, \tilde{\mathscr{H}}_{v}\right\}\right| \\
& \exp \left[\frac { i } { \hbar } \int \mathrm { d } t \left(\int _ { \Sigma _ { t } } \left(\pi^{i j} \partial_{t} h_{i j}-N \mathscr{H}_{T}\right.\right.\right. \\
& \left.\left.\left.-N^{i} \mathscr{H}_{i}+\varepsilon_{0} \lambda_{0}\right)-H_{\mathscr{B}_{t}}\right)\right] .
\end{aligned}
$$

Integration over the momentum $\pi^{i j}$ gives

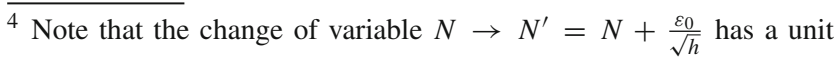
Jacobian, despite the fact that the transformation involves $\sqrt{h}$.
}

$$
\begin{aligned}
Z_{\mathrm{UG}}= & \mathscr{N}^{-1} \int \prod_{x^{\mu}} \mathscr{D} g_{\mu \nu} \mathscr{D} \lambda_{0} g^{00}(-g)^{-\frac{3}{2}}\left(N \int_{\Sigma_{t}} \sqrt{h}\right) \\
& \times \delta\left(\tilde{\chi}^{\mu}\right)\left|\operatorname{det}\left\{\tilde{\chi}^{\mu}, \tilde{\mathscr{H}}_{\nu}\right\}\right| \exp \left[\frac { i } { \hbar } \left(S_{\mathrm{EH}}\left[g_{\mu \nu}\right]\right.\right. \\
& \left.\left.-\int \mathrm{d} t \int_{\Sigma_{t}} \lambda_{0}\left(\sqrt{-g}-\varepsilon_{0}\right)\right)\right] .
\end{aligned}
$$

where $S_{\mathrm{EH}}\left[g_{\mu \nu}\right]$ is the Einstein-Hilbert action without a cosmological constant. Since the zero mode $\lambda_{0}$ depends only on time, integration over this variable gives a $\delta$-function that imposes the unimodular condition (1.2) to hold on each slice $\Sigma_{t}$ of spacetime in average,

$$
\begin{aligned}
Z_{\mathrm{UG}}= & \mathscr{N}^{-1} \int \prod_{x^{\mu}} \mathscr{D} g_{\mu \nu} g^{00}(-g)^{-\frac{3}{2}} \delta\left(\frac{\int_{\Sigma_{t}}\left(\sqrt{-g}-\varepsilon_{0}\right)}{N \int_{\Sigma_{t}} \sqrt{h}}\right) \\
& \times \delta\left(\tilde{\chi}^{\mu}\right)\left|\operatorname{det}\left\{\tilde{\chi}^{\mu}, \tilde{\mathscr{H}}_{\nu}\right\}\right| \exp \left(\frac{i}{\hbar} S_{\mathrm{EH}}\left[g_{\mu \nu}\right]\right) \cdot
\end{aligned}
$$

The integrated unimodular condition in the above path integral,

$\int_{\Sigma_{t}}\left(\sqrt{-g}-\varepsilon_{0}\right)=0$

does not constrain local deviations from the unimodular condition (1.2) as long as the average value of $\sqrt{-g}$ over $\Sigma_{t}$ remains fixed to that of $\varepsilon_{0}$. This is a quite surprising result, since we expected to see the unimodular condition to be imposed locally, like in the path integral for the HT action [14]. On the other hand, it makes some sense that quantum fluctuations around the classical field equation (1.2) are permitted. The physical purpose of the condition (4.38) is to ensure that the number of physical degrees of freedom in the path integral (4.37) matches that of DUG and GR, since together the gauge conditions $\tilde{\chi}^{\mu}$ and the condition (4.38) impose four conditions per point in space.

In this theory, the quantum effective action is a function of the perturbative gravitational field $f_{\mu \nu}$ which satisfies an integrated condition. Namely the trace of the perturbative field must have zero integral over $\Sigma_{t}$ at all times,

$\int_{\Sigma_{t}} f_{\mu}^{\mu}=0$

In other words, the quantum effective action is built in the same way as in the HT theory [14], except that the condition on the perturbative gravitational field $\left(f_{\mu}^{\mu}=0\right)$ is replaced with the integrated condition (4.39). The gravitational field is further constrained by the gauge conditions $\tilde{\chi}^{\mu}$.

\section{Counting of physical degrees of freedom}

In both cases, DUG and UG, Dirac's counting of physical degrees of freedom gives the same result: two propagating modes plus one zero/single mode. In DUG, the extra zero mode is the cosmological variable $\lambda$, which is a constant 
spatially and does not evolve. Thus the extra zero mode is not a true physical degrees of freedom. It is just a cosmological constant. Hence the physical degrees of freedom are the same as in GR.

In the UG theory with fixed metric determinant, the canonical structure is partially different from DUG. In particular the integral of the Hamiltonian constraint is a second class constraint, and hence the gauge/coordinate conditions must contain one zero mode less than in DUG and GR. This may appear to imply that the extra zero mode would be a physical degree of freedom, but our analysis shows otherwise. This is evident in the path integral (4.37), where the single $\delta$-function eliminates one zero mode by imposing the integrated unimodular condition (4.38). In other words it acts like an extra gauge/coordinate condition, so that the total number of conditions matches DUG and GR. Thus the actual number of physical degrees of freedom in UG is the same as in DUG and GR.

\section{Gauge fixing example}

We can choose the gauge conditions, for example, as

$$
\begin{aligned}
\bar{\chi}_{\mathrm{U}}^{0} & =\overline{\sqrt{h}(\ln h-\Phi)} \\
& =\sqrt{h}\left(\ln h-\Phi-\frac{1}{\int_{\Sigma_{t}} \sqrt{h}} \int_{\Sigma_{t}} \sqrt{h}(\ln h-\Phi)\right) \approx 0 \\
\chi_{\mathrm{U}}^{i} & =\partial_{j}\left(\sqrt{h} h^{i j}\right) \approx 0
\end{aligned}
$$

where $\Phi$ is a fixed function, and we denote the conditions collectively as $\tilde{\chi}_{\mathrm{U}}^{\mu}=\left(\bar{\chi}_{\mathrm{U}}^{0}, \chi_{\mathrm{U}}^{i}\right)$. The first gauge condition $\bar{\chi}_{\mathrm{U}}^{0}$ fixes the average free component of $\ln h$. That is the average free component of the first condition of the Faddeev-Popov gauge [33]. The gauge conditions $\chi_{\mathrm{U}}^{i}$ are the harmonic conditions on the spatial hypersurface.

We define an operator $Q_{\mathrm{U} v}^{\mu}$ in terms of the gauge transformation of the gauge conditions (4.40) as

$Q_{\mathrm{U} \nu}^{\mu} \tilde{\xi}^{\nu}=\left\{\tilde{\chi}_{\mathrm{U}}^{\mu}, \int_{\Sigma_{t}} \tilde{\mathscr{H}}_{\nu} \tilde{\xi}^{\nu}\right\}, \quad \tilde{\xi}^{\nu}=\left(\bar{\xi}, \xi^{i}\right)$

We obtain the components of the operator as

$$
\begin{aligned}
Q_{\mathrm{U} 0}^{0}= & 2 \sqrt{h} K+\bar{\chi}_{\mathrm{U}}^{0} K, \\
Q_{\mathrm{U} i}^{0}= & 2 \sqrt{h} D_{i}+\bar{\chi}_{\mathrm{U}}^{0} D_{i}, \\
Q_{\mathrm{U} 0}^{i}= & -2 \sqrt{h}\left(K^{i j}-\frac{1}{2} h^{i j} K\right) \partial_{j} \\
& -2 \partial_{j}\left[\sqrt{h}\left(K^{i j}-\frac{1}{2} \sqrt{h} h^{i j} K\right)\right], \\
Q_{\mathrm{U} j}^{i}= & -\sqrt{h} \delta_{j}^{i} h^{k l} \partial_{k} \partial_{l}-\delta_{j}^{i} \chi_{\mathrm{U}}^{k} \partial_{k}+\chi_{\mathrm{U}}^{i} \partial_{j}+\partial_{j} \chi_{\mathrm{U}}^{i} .
\end{aligned}
$$

The components (4.42)-(4.45) of the operator could be simplified by using the constraints and in particular the gauge conditions. Finally, the path integral is written as

$$
\begin{aligned}
Z_{\mathrm{UG}}= & \mathscr{N}^{-1} \int \prod_{x^{\mu}} \mathscr{D} g_{\mu \nu} \mathscr{D} \bar{\eta} \mathscr{D} \eta_{i} \mathscr{D} \bar{c}^{*} \mathscr{D} \bar{c} \mathscr{D} c_{i}^{*} \mathscr{D} c^{i} g^{00}(-g)^{-\frac{3}{2}} \\
& \times \delta\left(\frac{\int_{\Sigma_{t}}\left(\sqrt{-g}-\varepsilon_{0}\right)}{N \int_{\Sigma_{t}} \sqrt{h}}\right) \exp \left[\frac { i } { \hbar } \left(S_{\mathrm{EH}}\left[g_{\mu \nu}\right]\right.\right. \\
& -\int_{\mathscr{M}} \mathrm{d}^{4} x\left(\bar{\eta} \bar{\chi}_{\mathrm{U}}^{0}+\eta_{i} \chi_{\mathrm{U}}^{i}+\bar{c}^{*} Q_{\mathrm{U} 0}^{0} \bar{c}+\bar{c}^{*} Q_{\mathrm{U} i}^{0} c^{i}\right. \\
& \left.\left.\left.+c_{i}^{*} Q_{\mathrm{U} 0}^{i} \bar{c}+c_{i}^{*} Q_{\mathrm{U} j}^{i} c^{j}\right)\right)\right]
\end{aligned}
$$

where we have introduced pairs of anti-commuting ghosts $\bar{c}, \bar{c}^{*}$ and $c^{i}, c_{j}^{*}$, and auxiliary fields $\bar{\eta}, \eta_{i}$ for each gauge condition. The fields $\bar{c}, \bar{c}^{*}, \bar{\eta}$ have vanishing average over space, since they are associated with the generator $\overline{\mathscr{H}}_{T}$ and the gauge condition $\bar{\chi}_{\mathrm{U}}^{0}$. Including matter fields and defining the generating functional can be done similarly as in (3.83). Evidently, the above expression for the path integral is not covariant. The presence of integration over space in both the averaged unimodular condition and the definition of average free fields renders the expression noncovariant.

It indeed appears to be impossible to cast the path integral (4.37) into a fully covariant form. The underlying reason is the fact that the zero mode of the super-Hamiltonian is a second class constraint, and hence one of the gauge conditions must be average free over space. In order to achieve a covariant description, we have to enlarge the gauge symmetry so that the total super-Hamiltonian becomes a gauge generator. This was achieved in Sect. 3, where a generally covariant form of unimodular gravity is considered.

\section{The canonical relation of the two theories}

In the case with a fixed metric determinant, it is crucial to notice that the Hamiltonian (4.3) is not a constraint, since it contains the term $-\int_{\Sigma_{t}} \mathrm{~d}^{3} x \varepsilon_{0} \lambda$. Therefore the bulk part of the Hamiltonian does not vanish on the constraint surface. This is a striking difference compared to Hamiltonian of the fully diffeomorphism-invariant theory (3.20), which is a sum of first class constraints. However, there exists a clear relation between these Hamiltonians, since the nonvanishing term can be eliminated (or introduced) via a simple time-dependent canonical transformation.

Consider the following two canonical transformations of the variable $p_{\lambda} \rightarrow p_{\lambda}^{\prime}$ :

$p_{\lambda}=p_{\lambda}^{\prime} \pm \varepsilon_{0} t$,

with all other variables remaining unchanged. These two transformations are generated by the functionals

$$
F_{ \pm}=\int_{\Sigma_{t}} \mathrm{~d}^{3} x\left(\lambda p_{\lambda}^{\prime} \pm \varepsilon_{0} \lambda t\right)
$$


respectively. The Hamiltonian transforms to

$H^{\prime}=H+\frac{\partial F_{ \pm}}{\partial t}=H \pm \int_{\Sigma_{t}} \mathrm{~d}^{3} x \varepsilon_{0} \lambda$.

We can see that the transformation generated by $F_{+}$eliminates the nonvanishing term from the Hamiltonian (4.3), while the transformation generated by $F_{-}$introduces the nonvanishing term into the Hamiltonian (3.20). Notice that the variable $p_{\lambda}$ appears only in the primary constraints $\mathscr{C}_{\lambda} \approx 0$ and $p_{\lambda} \approx 0$ of the two theories, and these constraints drop out of the Hamiltonian due to the consistency conditions for their Lagrange multipliers (3.35) and (4.14), respectively.

The theory with fixed metric determinant can be shown to be a (partially) gauge fixed version of the fully diffeomorphism-invariant theory. When we introduce the following gauge fixing conditions into the Hamiltonian (3.48): ${ }^{5}$

$\mathscr{U}=N \sqrt{h}-\varepsilon_{0} \approx 0, \quad p_{\lambda}^{0} \approx 0, \quad \bar{p}_{\lambda} \approx 0, \quad V^{i} \approx 0$,

and together with the second class constraints $\mathscr{C}_{\lambda} \approx 0$ and $p_{n} \approx 0$, we obtain a Hamiltonian that has the same form as (4.28), except for the extra nonvanishing term in (4.28), $-\int_{\Sigma_{t}} \mathrm{~d}^{3} x \varepsilon_{0} \lambda_{0}$ with $\lambda_{0}$ given in (4.27). That extra term can be introduced with the canonical transformation (5.1) of the variable $p_{\lambda}$. Thus the theory (2.6) is a (partially) gauge fixed version of the theory (2.13). In other words, the fully diffeomorphism-invariant theory defined in (2.13) (and analyzed in Sect. 3) is a generalization of the unimodular theory of gravity with an enlarged gauge symmetry.

\section{Conclusions}

We have studied path integral quantization of two versions of unimodular gravity. In the fully diffeomorphism-invariant theory defined by the action (2.13), the path integral has the same form as the one of GR with a cosmological constant $\Lambda$ (3.82), except that the value of $\Lambda$ is not set by the action. The cosmological constant $\Lambda$ is an unspecified value of the variable $\lambda$. There exist two approaches regarding the interpretation of $\Lambda$ in this theory:

(i) The value of $\Lambda$ can be set in the boundary conditions of the path integral, since it is a boundary value of the variable $\lambda$. In this case, the value of $\Lambda$ is completely unspecified by the theory, and hence it needs to be set to the desired value by hand. One can use anthropic arguments for limiting the range of possible values of $\Lambda$ (see [5-9] for reviews), but we do not consider such arguments here. Physically, it makes no difference whether the observed

\footnotetext{
5 The first two gauge conditions are associated with the first class constraints $\pi_{N} \approx 0$ and $\mathscr{H}_{0} \approx 0$ (the zero mode of $\mathscr{H}_{T} \approx 0$ ), respectively.
}

value of $\Lambda$ is fixed by the boundary conditions or by setting the value of a coupling constant in the Lagrangian. Thus this approach is physically equivalent to GR.

(ii) Since the value of $\Lambda$ is unspecified, the vacuum state of the universe can be defined as a superposition of vacuum states corresponding to different values of $\Lambda$ [5]. Such an approach was used in $[12,13]$ where the path integral of the form (3.84) was conjectured. A similar path integral was later obtained in [14]. Starting from the action (2.13), we have derived the path integral (3.84) without any addition or manipulation of variables. The integration over $\Lambda$ arises due to the definition of the vacuum state (3.87). The given theory shows that it is unnecessary to impose the unimodular condition on the metric determinant in order to obtain the path integral (3.84). Using the semiclassical approximation and the stationary phase approximation one can argue [14] that the path integral (3.84) is dominated by the values of $\Lambda$ around the average energy density of matter over spacetime (3.90). It is presumed that the given values of $\Lambda$ were included in the vacuum state (3.87). This result is interesting but problematic. In order to estimate the average energy density of matter over spacetime, we need information on both the matter and the gravitational (background) fields, which depend on the assumed value of $\Lambda$. It could be interesting to search for alternative mechanisms that would single out the most likely values of $\Lambda$ within the fully diffeomorphism-invariant theory.

In the more conventional case defined by the action (2.6), the path integral (4.37) differs from the path integral of GR in two ways: (i) since the zero mode of the super-Hamiltonian (4.17) is a second class constraint, the first class Hamiltonian constraint (4.18) and an associated gauge condition have zero average over space, and (ii) the metric in the path integral must satisfy the integrated unimodular condition (4.38). The condition (4.38) imposes the unimodular condition (1.2) to hold in average over space at each moment in time. The path integral has a generally noncovariant form due to the given differences. The perturbative gravitational field in the (semiclassical) quantum effective action must satisfy the integrated condition (4.39).

At quantum level the unimodular condition can manifest itself in three ways. In the HT theory [3], the unimodular condition is imposed locally in the path integral and in the quantum effective action [14]. In the path integral and the quantum effective action of the UG theory (2.6), the unimodular condition is averaged over space (4.38). Lastly, the DUG theory (2.13) does not involve a unimodular condition.

In Sect. 5, we established the canonical relation of the two considered versions of unimodular gravity. While the actions (2.6) and (2.13) are shown to be equivalent classically, the time-dependent canonical transformation (5.1) involved in 
the relation of their Hamiltonian structures has an interesting effect to the quantum theory. That is the appearance of the averaged unimodular condition (4.38) in the path integral of UG (4.46). Furthermore, the gauge symmetry is restricted, since the integral of the super-Hamiltonian over space (4.17) becomes a second class constraint. This implies that the path integral involves a pair of ghost fields and a Lagrange multiplier field whose average values over space must vanish.

In practice, both of these implications are inconvenient to work with. Thus the fully diffeomorphism-invariant theory considered in Sect. 3, or the previously worked out HT theory, are the preferable versions of unimodular gravity for quantization.

The differences in the path integrals of different versions of unimodular gravity do not necessarily imply that the physical predictions of the theories are different. The DUG and HT theories can indeed be expected to be physically equivalent, since the theories are related by a simple change of an auxiliary variable (see below (2.13)). However, in addition to gauge fixing, the canonical relation between DUG and UG involves the time-dependent canonical transformation (5.1), which leads to the aforementioned complications. Therefore it is still unclear whether the path integrals (3.82) and (4.46) produce equivalent predictions. Confirming this would require the formulation of Feynman rules and the calculation of the scattering matrices. This is a very demanding task in itself, which we wish to investigate in further work.

Proper quantization of gravity requires more advanced methods. Two known approaches are the spin foam models and the dynamical triangulations. Some steps toward loop quantization of unimodular gravity have already been taken in [26], and more recently in [20].

Acknowledgments We are grateful to M. Chaichian for discussions and suggestions. R.B. thanks FAPESP for full support, Project No. 2013/26571-4. M.O. thankfully acknowledges support from the Emil Aaltonen Foundation. The support of the Academy of Finland under the Projects No. 136539 and 272919 is gratefully acknowledged.

Open Access This article is distributed under the terms of the Creative Commons Attribution 4.0 International License (http://creativecomm ons.org/licenses/by/4.0/), which permits unrestricted use, distribution, and reproduction in any medium, provided you give appropriate credit to the original author(s) and the source, provide a link to the Creative Commons license, and indicate if changes were made. Funded by SCOAP ${ }^{3}$.

\section{Appendix A: Quantization of gauge theories with linearly dependent generators}

The unimodular theories of gravity involve certain local gauge generators whose integrals over the spatial hypersurface vanish by definition. This type of a spatially nonlocal linear dependence of generators is an inherent feature of the unimodular gravity theories, where the cosmological constant appears as a (constant) value of a scalar variable. Quantization of gauge theories with linearly dependent generators was achieved in [28]. The Batalin-Vilkovisky formalism [28] is suitable for the description of the nonlocally linearly dependent generators of unimodular gravity. In particular, the average free nature of the Faddeev-Popov ghosts and auxiliary fields associated with the average free generators is explained naturally within the given formalism.

First we review the Batalin-Vilkovisky formalism. The formalism was applied to the minisuperspace formulation of Friedman-Robertson-Walker cosmology models in [29], where a review of the formalism for theories with only bosonic gauge fields is also presented. Since the gravitational sectors of the unimodular gravity theories involve only bosonic fields, our presentation follows [29] with a few conventional differences due to the following application to the unimodular gravity theories considered in Sects. 3 and 4.

When the generators $G_{\alpha}$ are linearly dependent, there exist right zero eigenvectors $Z_{a}^{\alpha}$,

$G_{\alpha} Z_{a}^{\alpha}=0$.

Here the condensed index $\alpha$ labels each local generator at every point in the spatial hypersurfaces. Hence summing over such an index involves an integration over space in addition to a sum over the components. ${ }^{6}$ The Latin index labels the zero eigenvectors $a=1, \ldots, A$. Here the vectors $Z_{a}^{\alpha}$ are linearly independent, i.e., we consider a first-stage reducible theory. The gauge conditions $\chi^{\alpha}$ have to be similarly redundant as the generators, so that there exist left zero eigenvectors $\hat{Z}_{\alpha}^{a}$,

$\hat{Z}_{\alpha}^{a} \chi^{\alpha}=0$.

The eigenvectors $Z_{a}^{\alpha}$ and $\hat{Z}_{\alpha}^{a}$ are the right and left zero vectors of the degenerate Faddeev-Popov operator,

$Q_{\beta}^{\alpha}=\left\{\chi^{\alpha}, G_{\beta}\right\}$,

respectively. Thus, within this formalism, the FaddeevPopov ghosts $c^{\alpha}, c_{\alpha}^{*}$ become gauge fields that require additional gauge fixing. For that purpose the set of Lagrange multipliers and ghosts $\left(c^{\alpha}, c_{\alpha}^{*}, \eta_{\alpha}\right)$ is extended to [28]

$\Phi_{\mathrm{g}}=\left(c^{\alpha}, c_{\alpha}^{*}, \eta_{\alpha}, C^{a}, C_{a}^{*}, E^{a}, \theta_{a}, \vartheta^{a}\right)$,

where $c^{\alpha}, c_{\alpha}^{*}, \theta_{a}, \vartheta^{a}$ are Grassmann anti-commuting variables and the rest are commuting variables. The path integral and the corresponding effective gauge fixed action are written as

\footnotetext{
$\overline{6}$ Unlike in [29] the sum over a condensed index does not involve integration over time. Furthermore we do not consider Euclidean quantum gravity, i.e., Wick rotation of time is not performed.
} 


$$
\begin{aligned}
Z= & \int \mathscr{D} \phi^{i} \mathscr{D} \pi_{i} \mathscr{D} \Phi_{\mathrm{g}} \exp \left(\frac{i}{\hbar} S_{\mathrm{eff}}\right), \\
S_{\mathrm{eff}}= & S-\int \mathrm{d} t\left[c_{\alpha}^{*} Q^{\alpha}{ }_{\beta} c^{\beta}+C_{a}^{*}\left(\omega_{\alpha}^{a} Z_{b}^{\alpha}\right) C^{b}\right. \\
& \left.+\eta_{\alpha}\left(\chi^{\alpha}+\sigma_{a}^{\alpha} E^{a}\right)+\theta_{a} \omega_{\alpha}^{a} c^{\alpha}+c_{\alpha}^{*} \sigma_{a}^{\alpha} \vartheta^{a}\right],
\end{aligned}
$$

where $\phi^{i}$ and $\pi_{i}$ are the gauge fields and their canonically conjugated momenta, and $S$ is the action without gauge fixing. The extra Lagrange multipliers $\left(\theta_{a}, \vartheta^{a}\right)$ impose the gauge conditions $\omega_{\alpha}^{a} c^{\alpha}$ and $c_{\alpha}^{*} \sigma_{a}^{\alpha}$ on the Faddeev-Popov ghosts, where the gauge parameters $\left(\omega_{\alpha}^{a}, \sigma_{a}^{\alpha}\right)$ are arbitrary. The variables $C_{a}^{*}$ and $C^{a}$ are the ghosts for the FaddeevPopov ghost fields. The so-called extra ghosts $E^{a}$ regulate divergent factors $\delta(0)$ that appear in the original gauge fixing $\delta\left(\chi^{\alpha}\right)$ with a redundant set of gauge conditions (A.2).

Integration over the ghost sector gives the path integral as

$$
\begin{aligned}
Z= & \int \mathscr{D} \phi^{i} \mathscr{D} \pi_{i} \frac{\operatorname{det} \mathscr{F}_{\beta}^{\alpha}}{\operatorname{det} q_{b}^{a} \operatorname{det} \hat{q}_{b}^{a}} \int \mathscr{D} E^{a} \delta\left(\chi^{\alpha}+\sigma_{a}^{\alpha} E^{a}\right) \\
& \times\left(\operatorname{det} \hat{q}_{b}^{a}\right) \exp \left(\frac{i}{\hbar} S\right)
\end{aligned}
$$

where the gauge fixed Faddeev-Popov operator is defined as

$$
\mathscr{F}_{\beta}^{\alpha}=Q_{\beta}^{\alpha}+\sigma_{a}^{\alpha} \omega_{\beta}^{a},
$$

and the following matrices are introduced:

$q_{b}^{a}=\omega_{\alpha}^{a} Z_{b}^{\alpha}, \quad \hat{q}_{b}^{a}=\hat{Z}_{\alpha}^{a} \sigma_{b}^{\alpha}$.

The path integral (A.6) is independent of the chosen gauge parameters $\left(\omega_{\alpha}^{a}, \sigma_{a}^{\alpha}\right)$, since both the ratio of determinants (det $\mathscr{F}_{\beta}^{\alpha} / \operatorname{det} q_{b}^{a} \operatorname{det} \hat{q}_{b}^{a}$ ) and the regulated gauge fixing factor are invariant under a change of the gauge parameters (see [29] for a proof).

Next we apply this formalism to the quantization of the two unimodular gravity theories (DUG and UG).

\section{A.1 Fully diffeomorphism-invariant unimodular gravity}

Let us consider the quantization of DUG presented in Sect. 3. The second class constraints are given in (3.58). The generators are

$G_{\alpha}=\left[\pi_{N}, \pi_{i}, p_{i}, \mathscr{H}_{T}^{\prime}, \mathscr{H}_{i}, \overline{\mathscr{C}}^{\prime}\right]$

with (3.59). Gauge fixing conditions are chosen as in (3.60),

$\chi^{\alpha}=\left[\sigma^{0}, \sigma^{i}, V^{i}, \chi^{0}, \chi^{i}, \bar{p}_{\lambda}\right]$

The generator $\overline{\mathscr{C}}^{\prime}$ and the corresponding gauge condition $\bar{p}_{\lambda}$ exhibit a nonlocal linear dependence, since their integrals over space vanish by definition. Hence there exist a single right zero vector,

$$
\begin{aligned}
Z^{\alpha} & =\left[0,0,0,0,0, \frac{\sqrt{h}}{\int_{\Sigma_{t}} \mathrm{~d}^{3} x \sqrt{h}}\right], \\
G_{\alpha} Z^{\alpha} & =\frac{1}{\int_{\Sigma_{t}} \mathrm{~d}^{3} x \sqrt{h}} \int_{\Sigma_{t}} \mathrm{~d}^{3} x \sqrt{h} \overline{\mathscr{C}}^{\prime}=0,
\end{aligned}
$$

and a single left zero vector,

$$
\begin{aligned}
\hat{Z}_{\alpha} & =[0,0,0,0,0,1], \\
\hat{Z}_{\alpha} \chi^{\alpha} & =\int_{\Sigma_{t}} \mathrm{~d}^{3} x \bar{p}_{\lambda}=0 .
\end{aligned}
$$

Since only one pair of zero vectors exist, we have dropped the label $a$ from the zero vectors and also from the other variables of the path integral (A.5).

We choose the gauge fixing parameters for the ghosts as

$\omega_{\alpha}=[0,0,0,0,0,-1]$,

$\sigma^{\alpha}=\left[0,0,0,0,0, \frac{\sqrt{h}}{\int_{\Sigma_{t}} \mathrm{~d}^{3} x \sqrt{h}}\right]$.

Thus the ghost fields $\left(\bar{c}, \bar{c}^{*}\right)$ associated with the generator $\overline{\mathscr{C}}^{\prime}$ are imposed to satisfy the gauge conditions

$\omega_{\alpha} c^{\alpha}=-\int_{\Sigma_{t}} \mathrm{~d}^{3} x \bar{c}=0$,

$c_{\alpha}^{*} \sigma^{\alpha}=\frac{1}{\int_{\Sigma_{t}} \mathrm{~d}^{3} x \sqrt{h}} \int_{\Sigma_{t}} \mathrm{~d}^{3} x \sqrt{h} \bar{c}^{*}=0$.

We obtain the (now one-dimensional) matrices (A.8) as

$q=\omega_{\alpha} Z^{\alpha}=-1, \quad \hat{q}=\hat{Z}_{\alpha} \sigma^{\alpha}=1$.

In the amended Faddeev-Popov operator (A.7), the gauge fixing term with (A.13) contributes to the part of $c_{\alpha}^{*} \mathscr{F}_{\beta}^{\alpha} c^{\beta}$ that involves the ghosts $\bar{c}, \bar{c}^{*}$ as

$$
\begin{aligned}
& \int_{\Sigma_{t}} \mathrm{~d}^{3} x d^{3} y \bar{c}^{*}(x)\left\{\bar{p}_{\lambda}(x), \overline{\mathscr{C}}^{\prime}(y)\right\} \bar{c}(y)+c_{\alpha}^{*} \sigma^{\alpha} \omega_{\beta} c^{\beta} \\
& =-\int_{\Sigma_{t}} \mathrm{~d}^{3} x \bar{c}^{*} \bar{c}
\end{aligned}
$$

This implies a unit contribution to the canonical measure of the path integral.

The gauge condition $\bar{p}_{\lambda}=0$ imposes $p_{\lambda}$ to become proportional to a spatial constant $p_{\lambda}^{0}(3.40)$, which is the integrated value of $p_{\lambda}$ over space (3.41). The extra ghost $E$ introduces an independent term into this gauge condition, so that the integral of the condition over space no longer vanishes,

$\hat{Z}_{\alpha}\left(\chi^{\alpha}+\sigma^{\alpha} E\right)=E$,

which serves as a regulator for the corresponding $\delta$-function in the path integral. The functional integral over $E$ forces the Lagrange multiplier of the gauge condition $\bar{p}_{\lambda}$ to have vanishing average value over space, 


$$
\begin{aligned}
\int \mathscr{D} \eta \mathscr{D} E \exp \left[\frac{i}{\hbar} \int_{\mathscr{M}} \mathrm{d}^{4} x \eta\left(\bar{p}_{\lambda}+\frac{\sqrt{h}}{\int_{\Sigma_{t}} \mathrm{~d}^{3} x \sqrt{h}} E\right)\right. & \omega_{\alpha}=\left[0, \frac{\sqrt{h}}{\int_{\Sigma_{t}} \mathrm{~d}^{3} x \sqrt{h}}, 0\right], \\
\propto \int \mathscr{D} \eta \delta\left(\frac{\int_{\Sigma_{t}} \mathrm{~d}^{3} x \sqrt{h} \eta}{\int_{\Sigma_{t}} \mathrm{~d}^{3} x \sqrt{h}}\right) \exp \left(\frac{i}{\hbar} \int_{\mathscr{M}} \mathrm{d}^{4} x \eta \bar{p}_{\lambda}\right), & \sigma^{\alpha}=\left[0, \frac{\sqrt{h}}{\int_{\Sigma_{t}} \mathrm{~d}^{3} x \sqrt{h}}, 0\right],
\end{aligned}
$$

which prevents the appearance of divergent factors $\delta(0)$. In Sects. 3.3 and 4.3, every $\delta$-function for an average free constraint is regulated in this way, and we denote such $\delta$ functions simply as

$$
\int \mathscr{D} \bar{\eta} \exp \left(\frac{i}{\hbar} \int_{\mathscr{M}} \mathrm{d}^{4} x \bar{\eta} \bar{p}_{\lambda}\right)=\delta\left(\bar{p}_{\lambda}\right)
$$

where the auxiliary field $\bar{\eta}$ is now assumed to have vanishing average over space.

Once the additional gauge fixing (A.14) on the ghosts associated with the linearly dependent generators and gauge conditions is performed, and the $\delta$-functions of the average free constraints are regulated, it is easy see how the canonical path integral is obtained as (3.63) after the nonphysical variables in the canonical integration measure (3.62) have been integrated out (except for those variables deleted by the gauge conditions $\chi^{\mu}$ that are unspecified). In summary, the path integral obtained in Sect. 3.3 corresponds to the specific choice of the gauge parameters (A.13), which are responsible for the additional gauge fixing required by the linearly dependent generators.

\section{A.2 Unimodular gravity with a fixed metric determinant}

Here we consider the quantization of UG presented in Sect. 4. The generators are

$G_{\alpha}=\left[\pi_{i}, \overline{\mathscr{H}}_{T}^{\prime}, \mathscr{H}_{i}^{\prime}\right]$

with (4.31). Gauge conditions are chosen as in (4.32),

$\chi^{\alpha}=\left[\sigma^{i}, \bar{\chi}^{0}, \chi^{i}\right]$.

We again have a nonlocal linear dependence, since the integrals of $\overline{\mathscr{H}}_{T}^{\prime}$ and $\bar{\chi}^{0}$ over the spatial hypersurface vanish. A single pair of zero vectors is obtained as

$$
\begin{aligned}
& Z^{\alpha}=[0,1,0], \quad Z^{\alpha} G_{\alpha}=\int_{\Sigma_{t}} \mathrm{~d}^{3} x \overline{\mathscr{H}}_{T}^{\prime}=0, \\
& \hat{Z}_{\alpha}=[0,1,0], \quad \hat{Z}_{\alpha} \chi^{\alpha}=\int_{\Sigma_{t}} \mathrm{~d}^{3} x \bar{\chi}^{0}=0 .
\end{aligned}
$$

The gauge fixing parameters $\omega_{\alpha}$ and $\sigma^{\alpha}$ can be chosen so that the ghosts $\left(\bar{c}, \bar{c}^{*}\right)$ associated with the generator $\overline{\mathscr{H}}_{T}^{\prime}$ satisfy the condition of vanishing average value over space. The parameters are chosen as

and the gauge conditions on the ghosts read

$$
\begin{aligned}
& \omega_{\alpha} c^{\alpha}=\frac{1}{\int_{\Sigma_{t}} \mathrm{~d}^{3} x \sqrt{h}} \int_{\Sigma_{t}} \mathrm{~d}^{3} x \sqrt{h} \bar{c}=0, \\
& c_{\alpha}^{*} \sigma^{\alpha}=\frac{1}{\int_{\Sigma_{t}} \mathrm{~d}^{3} x \sqrt{h}} \int_{\Sigma_{t}} \mathrm{~d}^{3} x \sqrt{h} \bar{c}^{*}=0 .
\end{aligned}
$$

The determinants of the matrices (A.8) have unit values

$q=\omega_{\alpha} Z^{\alpha}=1, \quad \hat{q}=\hat{Z}_{\alpha} \sigma^{\alpha}=1$.

The second class constraints (4.30) contain a pair of average free constraints $\left(\bar{\lambda} \approx 0, \bar{p}_{\lambda} \approx 0\right.$ ), which have to be treated in a similar way as a nonlocally linearly dependent generator and a gauge condition. In the path integral, the contribution of these constraints is just a unit factor to the Faddeev-Popov determinant, which is quite similar to the case of the constraints $\overline{\mathscr{C}}^{\prime}$ and $\bar{p}_{\lambda}$ in DUG. Hence we shall omit the analysis of these constraints here.

Lastly, we explain how the path integral in Sect. 4.3 is obtained from the present formalism. As was discussed above, all the $\delta$-functions for average free constraints has to be regulated in order to avoid divergent $\delta(0)$ factors. Integration over the extra ghost $E$ and the additional Lagrange multipliers $(\theta, \vartheta)$ produces the $\delta$-functions that impose the Lagrange multiplier of the gauge condition $\bar{\chi}^{0}$ and the ghosts $\left(\bar{c}, \bar{c}^{*}\right)$ associated with the linearly dependent generators to become average free over the spatial hypersurface,

$\delta\left(\eta_{\alpha} \sigma^{\alpha}\right) \delta\left(\omega_{\alpha} c^{\alpha}\right) \delta\left(c_{\alpha}^{*} \sigma^{\alpha}\right)$,

where the conditions for the ghosts are (A.24) and the condition for the Lagrange multiplier $\bar{\eta}$ is

$\eta_{\alpha} \sigma^{\alpha}=\frac{1}{\int_{\Sigma_{t}} \mathrm{~d}^{3} x \sqrt{h}} \int_{\Sigma_{t}} \mathrm{~d}^{3} x \sqrt{h} \bar{\eta}=0$.

In Sect. 4.3, the $\delta$-functions (A.26) are omitted in the path integral (4.46), since the fields $\bar{\chi}^{0}, \bar{c}, \bar{c}^{*}$ are assumed to satisfy the conditions (A.24) and (A.27) from the beginning. The Faddeev-Popov determinant in the path integral is defined in terms of ghost fields that satisfy the conditions (A.24), so that no zero modes are present in the ghost sector that generates the determinant. In particular, the nontrivial part of the determinant in the path integral (4.34) (and thereafter) is written as ${ }^{7}$

\footnotetext{
$\overline{7}$ Note that here the sums over the repeated indices $\mu, v$ include integration over the spatial hypersurface.
} 


$$
\begin{aligned}
\left|\operatorname{det}\left\{\tilde{\chi}^{\mu}, \tilde{\mathscr{H}}_{v}\right\}\right|= & \int \mathscr{D} \tilde{c}_{\mu}^{*} \mathscr{D} \tilde{c}^{\mu} \\
& \times \exp \left(-\frac{i}{\hbar} \int \mathrm{d} t \tilde{c}_{\mu}^{*}\left\{\tilde{\chi}^{\mu}, \tilde{\mathscr{H}}_{\mu}\right\} \tilde{c}^{v}\right),
\end{aligned}
$$

where the first components of the ghost fields $\tilde{c}^{\mu}=\left(\bar{c}, c^{i}\right)$ and $\tilde{c}_{\mu}^{*}=\left(\bar{c}^{*}, c_{i}^{*}\right)$ are assumed to be average free over space. Finally, we can see that the path integral obtained in Sect. 4.3 corresponds to the specific choice of the gauge parameters (A.23), which are responsible for imposing the necessary conditions on the ghosts (A.24) and on the Lagrange multipliers (A.27).

\section{References}

1. A. Einstein, The foundation of the general theory of relativity. Ann. Phys. 49, 769 (1916). Translated and included in The Principle of Relativity by H.A. Lorentz et al. (Dover Press, New York, 1923)

2. A. Einstein, Do gravitational fields play an essential part in the structure of the elementary particles of matter? Sitzungsber. Preuss. Akad. Wiss. Berlin (Math. Phys.) 1919, 433 (1919). Translated and included in The Principle of Relativity, by H.A. Lorentz et al. (Dover Press, New York, 1923)

3. M. Henneaux, C. Teitelboim, The cosmological constant and general covariance. Phys. Lett. B 222, 195 (1989). doi:10.1016/ 0370-2693(89)91251-3

4. W.G. Unruh, A unimodular theory of canonical quantum gravity. Phys. Rev. D 40, 1048 (1989). doi:10.1103/PhysRevD.40.1048

5. S. Weinberg, The cosmological constant problem. Rev. Mod. Phys. 61, 1 (1989). doi:10.1103/RevModPhys.61.1

6. T. Padmanabhan, Cosmological constant: the weight of the vacuum. Phys. Rep. 380, 235 (2003). doi:10.1016/ S0370-1573(03)00120-0. arXiv:hep-th/0212290

7. S. Nobbenhuis, Categorizing different approaches to the cosmological constant problem. Found. Phys. 36, 613 (2006). doi:10.1007/ s10701-005-9042-8. arXiv:gr-qc/0411093

8. R. Bousso, TASI lectures on the cosmological constant. Gen. Relativ. Gravit. 40, 607 (2008). doi:10.1007/s10714-007-0557-5. arXiv:0708.4231 [hep-th]

9. C.P. Burgess, The cosmological constant problem: why it's hard to get dark energy from micro-physics. arXiv:1309.4133[hep-th]

10. A. Padilla, Lectures on the cosmological constant problem. arXiv:1502.05296 [hep-th]

11. A. Álvarez, S. González-Martín, M. Herrero-Valea, C.P. Martín, Unimodular gravity redux. Phys. Rev. D 92, 061502 (2015). doi: 10. 1103/PhysRevD.92.061502. arXiv:1505.00022 [hep-th]

12. Y.J. Ng, H. van Dam, Possible solution to the cosmological constant problem. Phys. Rev. Lett. 65, 1972 (1990). doi:10.1103/ PhysRevLett.65.1972

13. Y.J. Ng, H. van Dam, Unimodular theory of gravity and the cosmological constant. J. Math. Phys. 32, 1337 (1991). doi:10.1063/ 1.529283

14. L. Smolin, Quantization of unimodular gravity and the cosmological constant problems. Phys. Rev. D 80, 084003 (2009). doi:10. 1103/PhysRevD.80.084003. arXiv:0904.4841 [hep-th]
15. A.H. Chamseddine, V. Mukhanov, A. Vikman, Cosmology with mimetic matter. JCAP 1406, 017 (2014). doi:10.1088/1475-7516/ 2014/06/017. arXiv:1403.3961 [astro-ph.CO]

16. R.D. Sorkin, On the role of time in the sum over histories framework for gravity. Int. J. Theor. Phys. 33, 523 (1994). doi:10.1007/ BF00670514. Originally presented at the conference, The History of Modern Gauge Theories, held at Logan, Utah, July 1987

17. W.G. Unruh, R.M. Wald, Time and the interpretation of canonical quantum gravity. Phys. Rev. D 40, 2598 (1989). doi:10.1103/ PhysRevD.40.2598

18. K.V. Kuchar, Does an unspecified cosmological constant solve the problem of time in quantum gravity? Phys. Rev. D 43, 3332 (1991). doi:10.1103/PhysRevD.43.3332

19. A. Padilla, I.D. Saltas, A note on classical and quantum unimodular gravity. arXiv:1409.3573 [gr-qc]

20. L. Smolin, Unimodular loop quantum gravity and the problems of time. Phys. Rev. D 84, 044047 (2011). doi:10.1103/PhysRevD.84. 044047. arXiv:1008.1759 [hep-th]

21. W. Buchmuller, N. Dragon, Einstein gravity from restricted coordinate invariance. Phys. Lett. B 207, 292 (1988). doi:10.1016/ 0370-2693(88)90577-1

22. B. Fiol, J. Garriga, Semiclassical unimodular gravity. JCAP 1008, 015 (2010). doi:10.1088/1475-7516/2010/08/015. arXiv:0809.1371 [hep-th]

23. C. Lanczos, The Variational Principles of Mechanics, 4th edn. (University of Toronto Press, Toronto, 1970)

24. P.A.M. Dirac, Lectures on Quantum Mechanics (Yeshiva University, New York, 1964)

25. S.W. Hawking, G.T. Horowitz, The gravitational Hamiltonian, action, entropy and surface terms. Class. Quantum Gravity 13, 1487 (1996). doi:10.1088/0264-9381/13/6/017. arXiv:gr-qc/9501014

26. L. Bombelli, W.E. Couch, R.J. Torrence, Time as space-time four volume and the Ashtekar variables. Phys. Rev. D 44, 2589 (1991). doi:10.1103/PhysRevD.44.2589

27. J. Kluson, Canonical analysis of unimodular gravity. Phys. Rev. D 91, 064058 (2015). doi:10.1103/PhysRevD.91.064058. arXiv: 1409.8014 [hep-th]

28. I.A. Batalin, G.A. Vilkovisky, Quantization of Gauge theories with linearly dependent generators. Phys. Rev. D 28, 2567 (1983). doi:10.1103/PhysRevD.28.2567

29. A.O. Barvinsky, The path integral for the statistical sum of the microcanonical ensemble in cosmology. JCAP 1104, 034 (2011). doi:10.1088/1475-7516/2011/04/034. arXiv:1012.1568 [hep-th]

30. A. Chaharsough Shirazi, J. Engle, Purely geometric path integral for spin foams. Class. Quantum Gravity 31, 075010 (2014). doi:10. 1088/0264-9381/31/7/075010. arXiv:1308.2946 [gr-qc]

31. P.A.M. Dirac, Fixation of coordinates in the Hamiltonian theory of gravitation. Phys. Rev. 114, 924 (1959). doi:10.1103/PhysRev. 114.924

32. E.S. Fradkin, G.A. Vilkovisky, S matrix for gravitational field. II. Local measure, general relations, elements of renormalization theory. Phys. Rev. D 8, 4241 (1973). doi:10.1103/PhysRevD.8.4241

33. L.D. Faddeev, V.N. Popov, Covariant quantization of the gravitational field. Sov. Phys. Usp. 16, 777 (1974). doi:10.1070/ PU1974v016n06ABEH004089 